\title{
Status of rates and rate equations for thermal leptogenesis
}

S. Biondini`1 D. Bödeker ${ }^{\dagger}$, N. Brambilla ${ }^{\ddagger}$, M. Garny ${ }^{\ddagger}$, J. Ghiglieri ${ }^{\S}$,

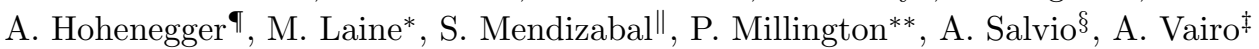

${ }^{*} A E C$, Institute for Theoretical Physics, University of Bern, Sidlerstrasse 5, CH-3012 Bern, Switzerland

${ }^{\dagger}$ Fakultät für Physik, Universität Bielefeld, 33501 Bielefeld, Germany

¥Physik-Department, Technische Universität München, James-Franck-Str. 1, 85748 Garching, Germany

${ }^{\S}$ CERN, Theoretical Physics Department, Geneva, Switzerland

"Faculty of Science and Technology, University of Stavanger, $N$-4036 Stavanger, Norway

"Department of Physics, Universidad Técnica Federico Santa María, Casilla 110-V, Valparaíso, Chile

${ }^{* *}$ School of Physics and Astronomy, University of Nottingham, Nottingham NG7 2RD, UK

\begin{abstract}
In many realizations of leptogenesis, heavy right-handed neutrinos play the main role in the generation of an imbalance between matter and antimatter in the early Universe. Hence, it is relevant to address quantitatively their dynamics in a hot and dense environment by taking into account the various thermal aspects of the problem at hand. The strong washout regime offers an interesting framework to carry out calculations systematically and reduce theoretical uncertainties. Indeed, any matter-antimatter asymmetry generated when the temperature of the hot plasma $T$ exceeds the right-handed neutrino mass scale $M$ is efficiently erased, and one can focus on the temperature window $T \ll M$. We review recent progresses in the thermal field theoretic derivation of the key ingredients for the leptogenesis mechanism: the right-handed neutrino production rate, the $\mathrm{CP}$ asymmetry in the heavy-neutrino decays and the washout rates. The derivation of evolution equations for the heavy-neutrino and lepton-asymmetry number densities, their rigorous formulation and applicability are also discussed.
\end{abstract}

\footnotetext{
${ }^{1}$ Corresponding Author.
} 


\section{Contents}

Status of rates and rate equations for thermal leptogenesis 1

1. Introduction . . . . . . . . . . . . . . . . . . . . . . . 2

2. Basics of thermal leptogenesis . . . . . . . . . . . . . . . . . . . 4

3. The right-handed neutrino production rate . . . . . . . . . . . . . . . . 9

3.1. In-vacuum and thermal corrections in the non-relativistic regime . . . . . . . . 9

3.2. Right-handed neutrino production rate in the relativistic regime . . . . . . . . . . 12

4. CP-violating parameter from right-handed neutrino decays . . . . . . . . . . . . . . 14

4.1. Towards NLO corrections to the CP-violating parameter in the non-relativistic regime ......................... 14

4.2. CP-violating parameter and lepton asymmetry with non-equilibrium quantum field

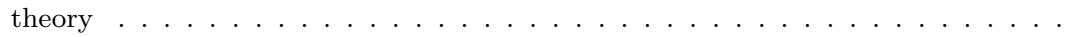

5. Different approaches to rate equations for particle dynamics in the early Universe . . 20

5.1. On the applicability and limitation of standard Boltzmann equations . . . . . . . 20

5.2. Effective kinetic equations and real-time correlation functions at finite temperature 23

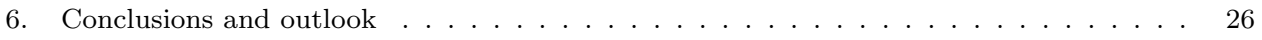

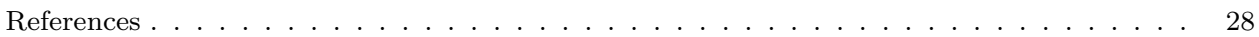

\section{Introduction}

The explanation of the observed baryon asymmetry of the Universe is an interesting and challenging endeavor for both cosmology and particle physics. Since any primordial imbalance between particles and antiparticles in the early Universe has likely been washed out after the inflationary epoch, a dynamical generation of the baryon asymmetry after the reheating phase appears favoured. Such dynamical generation in the context of quantum field theory is called baryogenesis.

One of the most attractive frameworks for baryogenesis is via leptogenesis [1]. In its original formulation, leptogenesis demands a modest extension of the Standard Model (SM): namely, the addition of right-handed $(\mathrm{RH})$ neutrinos with large Majorana masses far above the electroweak scale. The RH (or sterile) neutrinos are singlets under the SM gauge group, whereas they are minimally coupled to the SM particles via complex Yukawa couplings. The latter provide an additional source of $\mathrm{CP}$ violation with respect to the one already present in the quark sector of the SM. The Lagrangian of the model, when expressing the $\mathrm{RH}$ neutrinos in a basis where the mass matrix is diagonal and with Majorana fields, reads 1

$$
\mathcal{L}=\mathcal{L}_{\mathrm{SM}}+\frac{1}{2} \bar{N}_{k} i \not \partial N_{k}-\frac{M_{k}}{2} \bar{N}_{k} N_{k}-\left(\lambda_{\alpha k} \bar{\ell}_{\alpha} \phi^{c} N_{k}+\text { h.c. }\right)
$$

where $\mathcal{L}_{\mathrm{SM}}$ is the SM Lagrangian, $N_{k}$ are Majorana spinors $\left(N_{k}=N_{k}^{c}, N_{k}^{c}\right.$ being the charge-conjugate spinor of $N_{k}$ ) with mass $M_{k}$ and $k=1,2,3$ is the mass-eigenstate

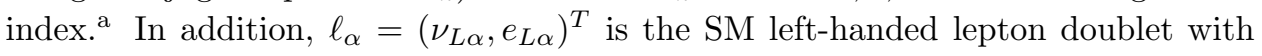
$\alpha=e, \mu, \tau, \phi^{c}=\epsilon \phi^{*}\left(\epsilon_{12}=-1\right)$ is the isospin-conjugate of the Higgs doublet, $\lambda_{\alpha k}$ are complex Yukawa couplings. We shall adopt the following notation for the

aThere exist other formulations of the very same Lagrangian where the field $N_{k}$ is understood as a two-component spinor, or alternatively, as a four-component spinor where only the right-handed entries are non-zero (see, e.g. 2]). They differ necessarily in the normalization of the kinetic terms. 
Yukawa couplings when summing over active lepton flavor: $\lambda_{i}^{*} \lambda_{k} \equiv \sum_{\alpha} \lambda_{\alpha i}^{*} \lambda_{\alpha k}$ (for $\left.k=i,\left|\lambda_{k}\right|^{2} \equiv \sum_{\alpha} \lambda_{\alpha k}^{*} \lambda_{\alpha k}\right)$.

In the standard picture, the $\mathrm{RH}$ neutrinos are produced by thermal scatterings in the early Universe and then decay out of equilibrium either into SM leptons or antileptons in different amounts due to the CP-violating phases. Such an asymmetry in the lepton sector is then partially converted into a baryon asymmetry by sphaleron processes in the SM [3].

Majorana neutrinos decay in a hot medium, namely the Universe in its early stages. Interactions with the medium modify the neutrino dynamics, through their production rate, masses and $\mathrm{CP}$ asymmetries, and affect the thermodynamic evolution of the lepton asymmetry. In order to take into account various thermal effects in leptogenesis properly, one has to consider the framework of quantum field theory at finite temperature. In so doing, all the ingredients in the analysis can be cast on a sound theoretical footing. However, the derivation of observables at finite temperature poses both conceptual and technical challenges. The purpose of the present paper is to review and report the recent results and developments on this subject.

We shall discuss thermal leptogenesis in the so-called strong washout regime. In the literature, the strong washout scenario is usually defined in terms of the decay parameter for the $k$-th $\mathrm{RH}$ neutrino, $K_{k}=\Gamma_{k} / H$ 4, 5, which is the ratio between the neutrino decay rate and the Hubble rate. The former is taken at $T=0$ and the latter is evaluated at $T=M_{k}$. For $K_{k}$ larger than one, the strong washout gets realized, enforcing decays and inverse decays to thermalize rapidly at $T \sim M_{k}$. Therefore, any initial lepton asymmetry possibly present before the onset of leptogenesis is erased [4,5] and the neutrino dynamics is close to equilibrium. The decay parameter can in turn be related to active neutrino mass parameters [5, 6], $K_{k}=\tilde{m}_{k} / m_{*}$, where $\tilde{m}_{k}=\left|\lambda_{k}\right|^{2} v^{2} /\left(2 M_{k}\right)$ (effective neutrino masses), $m_{*} \simeq 1.1 \times$ $10^{-3} \mathrm{eV}$ (equilibrium neutrino mass) and $v=246 \mathrm{GeV}$ is the vacuum expectation value of the SM Higgs field. By assigning to the effective neutrino masses either the solar or atmospheric neutrino mass scale from the available neutrino mixing and oscillations data 7,8 , the decay parameter is estimated to be $\mathcal{O}(K) \sim 10-$ 50. It is then conceivable that the strong washout regime was established in the early Universe. In this case, the dependence on the initial conditions is practically absent. Leptogenesis may then be highly predictive, and we can focus on deriving relevant observables for temperatures $T \ll M$.

The outline of the chapter is as follows. In Sec. 2, we provide an introduction to thermal leptogenesis and the rates involving $\mathrm{RH}$ neutrinos. In Sec. 3 , the neutrino production rate is expressed in terms of a neutrino thermal width and we discuss how in-vacuum and thermal corrections can be derived. Both the non-relativistic regime and the more challenging relativistic regime are addressed. Then we introduce the $\mathrm{CP}$ asymmetry in $\mathrm{RH}$ neutrino decays in Sec. 4. Thermal corrections to the $\mathrm{CP}$ asymmetry in the non-relativistic regime are reviewed, whose derivation 
are based on effective field theory (EFT) techniques. Moreover, their connection with the lepton asymmetry is discussed in the framework of non-equilibrium field theory. In Sec. 55, the rate equations governing the evolution of the heavy-neutrino and lepton-asymmetry number densities are described. In particular, we focus on the applicability of the Boltzmann equations for leptogenesis and on different approaches to their generalization in a quantum field theory framework. Conclusions and outlook are found in Sec. 6.

\section{Basics of thermal leptogenesis}

The sections that follow review the recent progress made in the calculation of the various $\mathrm{CP}$-even and -odd rates needed to obtain quantitatively accurate estimates of the final asymmetry in thermal leptogenesis. In order to motivate these discussions, we first outline the basics of thermal leptogenesis. Whilst the rest of this chapter will focus on first-principles and finite-temperature field-theoretic treatments, we will concentrate here on semi-classical treatments of leptogenesis, which involve supplementing systems of Boltzmann equations with the (zero-temperature) field-theoretic ingredients needed to capture the source(s) of CP violation. For more comprehensive overviews of the fundamentals of leptogenesis, see Refs. [4, 5, 8 10.

The simplest scenario of thermal leptogenesis is realised when the heavy-neutrino mass spectrum is hierarchical, i.e. $M_{1} \ll M_{2}<M_{3}$, and the decay rate of the lightest $\mathrm{RH}$ neutrino $\left(N_{1}\right)$ is larger than the Hubble rate, i.e. $\Gamma_{1} \gg H$. The dominant production of lepton asymmetry then occurs through the out-of-equilibrium decays of $N_{1}$ at temperatures $T \ll M_{1}$. Moreover, taking $M_{1} \gg 10^{12} \mathrm{GeV}$, charged-lepton flavor effects are unimportant (see, e.g., Ref. 8, 11] and Sec. 5.2) and the RH neutrinos are non-relativistic during the production of the asymmetry.

In order to estimate this asymmetry, we need to describe the evolution of the net lepton number $n_{\Delta \ell}$ (only the left-handed SM leptons are accounted for) and the number density of RH neutrinos $n_{N_{1}}$. We start with the coupled system of semiclassical Boltzmann equations for the phase-space distribution functions of the various species $f_{a}\left(t, \mathbf{X}_{a}, \mathbf{p}_{a}\right)$, whose single-particle energies are $\omega_{a}\left(\mathbf{p}_{a}\right)=\sqrt{\mathbf{p}_{a}^{2}+m_{a}^{2}}$. In a Friedmann-Lemaitre-Robertson-Walker Universe with Hubble rate $H$, there is no dependence on the spatial coordinate $\mathbf{X}_{a}$, and the semi-classical Boltzmann equations have the form (see, e.g., Refs. [5] and [12]):

$$
\begin{aligned}
\omega_{a} \frac{\partial f_{a}}{\partial t}-H \mathbf{p}_{a}^{2} \frac{\partial f_{a}}{\partial \omega_{a}}= & -\frac{1}{2} \sum_{a \mathcal{X} \leftrightarrow \mathcal{Y}} \int \mathrm{d} \Pi_{\mathcal{X}} \mathrm{d} \Pi_{\mathcal{Y}}(2 \pi)^{4} \delta^{4}\left(p_{a}+p_{\mathcal{X}}-p_{\mathcal{Y}}\right) \\
\times\left[f_{a} f_{\mathcal{X}} \mid \mathcal{M}(a \mathcal{X}\right. & \left.\rightarrow \mathcal{Y})\left.\right|^{2} F_{\mathcal{Y}}-f_{\mathcal{Y}}|\mathcal{M}(\mathcal{Y} \rightarrow a \mathcal{X})|^{2} F_{a} F_{\mathcal{X}}\right] .
\end{aligned}
$$

Our notation is as follows: $f_{\mathcal{A}}(t,\{\mathbf{p}\}) \equiv \prod_{i \in \mathcal{A}} f_{i}\left(t, \mathbf{p}_{i}\right)$ is the distribution function of the multi-particle initial state $\mathcal{A}=\mathcal{X}, \mathcal{Y} ; F_{\mathcal{B}}(t,\{\mathbf{p}\}) \equiv \prod_{i \in \mathcal{B}}\left(1 \pm f_{i}\left(t, \mathbf{p}_{i}\right)\right)$ contains the Bose-enhancement $(+)$ or Pauli-blocking factors $(-)$, due to the quantum statistics of the final states $\mathcal{B}=a, \mathcal{X}, \mathcal{Y} ; \mathcal{M}(a \mathcal{X} \rightarrow \mathcal{Y})$ is the matrix element for the 
process $a \mathcal{X} \rightarrow \mathcal{Y} ; p_{\mathcal{A}}^{\mu}=\sum_{i \in \mathcal{A}} p_{i}^{\mu} ;$ and $\mathrm{d} \Pi_{\mathcal{A}} \equiv \sigma_{\mathcal{A}} \prod_{i \in \mathcal{A}} \frac{\mathrm{d}^{4} p_{i}}{(2 \pi)^{4}} 2 \pi \theta\left(p_{i}^{0}\right) \delta\left(p_{i}^{2}-m_{i}^{2}\right)$ is the Lorentz-invariant phase-space measure, in which we include a symmetry factor $\sigma_{\mathcal{A}}$.

The semi-classical Boltzmann equations above can be recast in a simpler form by making the assumptions of: (i) kinetic (but not chemical) equilibrium and (ii) Maxwell-Boltzmann statistics (valid for the non-relativistic RH neutrinos and leading to a $10 \%$ error for the relativistic species 12 ). These approximations allow us to assume classical statistics, wherein $F_{\mathcal{B}} \approx 1$, and the distribution functions can be written in the form

$$
f_{a}\left(t, \mathbf{p}_{a}\right)=\frac{n_{a}(t)}{n_{a}^{\mathrm{eq}}} e^{-\omega_{a}\left(\mathbf{p}_{a}\right) / T}, \quad n_{a}(t)=g_{a} \int \frac{\mathrm{d}^{3} \mathbf{p}_{a}}{(2 \pi)^{3}} f_{a}\left(t, \mathbf{p}_{a}\right),
$$

where the number density $n_{a}(t)$ has the equilibrium form

$$
n_{a}^{\mathrm{eq}}=g_{a} \int \frac{\mathrm{d}^{3} \mathbf{p}_{a}}{(2 \pi)^{3}} e^{-\omega_{a}\left(\mathbf{p}_{a}\right) / T}=\frac{g_{a} z_{a}^{2} T^{3}}{2 \pi^{2}} \mathcal{K}_{2}\left(z_{a}\right) .
$$

The factor $g_{a}$ counts the number of degenerate internal degrees of freedom, $z_{a} \equiv$ $m_{a} / T$ and $\mathcal{K}_{n}$ is the $n$-th order modified Bessel function of the second kind. We are now able to integrate over the Lorentz-invariant phase space of the species $a$ to obtain the thermally-averaged Boltzmann equation

$$
\dot{n}_{a}(t)+3 H n_{a}(t)=-\sum_{a \mathcal{X} \leftrightarrow \mathcal{Y}}\left[\frac{n_{a} n_{\mathcal{X}}}{n_{a}^{\mathrm{eq}} n_{\mathcal{X}}^{\mathrm{eq}}} \gamma(a \mathcal{X} \rightarrow \mathcal{Y})-\frac{n_{\mathcal{Y}}}{n_{\mathcal{Y}}^{\mathrm{eq}}} \gamma(\mathcal{Y} \rightarrow a \mathcal{X})\right],
$$

where the $\gamma(a \mathcal{X} \rightarrow \mathcal{Y})$ are the thermally-averaged rates:

$$
\gamma(a \mathcal{X} \rightarrow \mathcal{Y})=\int \mathrm{d} \Pi_{a} \mathrm{~d} \Pi_{\mathcal{X}} \mathrm{d} \Pi_{\mathcal{Y}}(2 \pi)^{4} \delta^{4}\left(p_{a}+p_{\mathcal{X}}-p_{\mathcal{Y}}\right) f_{a}^{\text {eq }} f_{\mathcal{X}}^{\text {eq }}|\mathcal{M}(a \mathcal{X} \rightarrow \mathcal{Y})|^{2}
$$

The relevant processes for the $N_{1}$-dominated scenario of thermal leptogenesis are the decays and inverse decays of the lightest $\mathrm{RH}$ neutrino, as well as the leptonnumber-violating scattering processes that it mediates.

In the radiation era, the cosmic time $t$ can be related to the variable $z \equiv M_{1} / T$ via $t=z^{2} /\left(2 H_{N}\right)$, where $H_{N} \equiv H(z=1)$. We can then recast the rate equations in still simpler form by introducing the yields

$$
Y_{N_{1}}=\frac{n_{N_{1}}}{s} \quad \text { and } \quad Y_{\Delta \ell}=\sum_{\alpha} Y_{\Delta \ell_{\alpha}}=\sum_{\alpha} \frac{n_{\ell_{\alpha}}-\bar{n}_{\ell_{\alpha}}}{s}
$$

where $s=2 \pi^{2} g_{*} T^{3} / 45$ is the entropy density of the $g_{*}$ effective degrees of freedom. For the heavy-neutrino yield, the evolution is dominated by decays and inverse decays, and we obtain the rate equation

$$
\frac{s H_{N}}{z} \frac{\mathrm{d} Y_{N_{1}}}{\mathrm{~d} z}=-\left(\frac{Y_{N_{1}}}{Y_{N_{1}}^{\mathrm{eq}}}-1\right) \sum_{\alpha} \gamma_{\ell_{\alpha} \phi}^{N_{1}},
$$

where we have defined the CP-even rates $\gamma_{\mathcal{Y}} \equiv \gamma(\mathcal{X} \rightarrow \mathcal{Y})+\gamma(\overline{\mathcal{X}} \rightarrow \overline{\mathcal{Y}})$. For the total lepton asymmetry, we must consider both the heavy-neutrino decays and 
inverse decays, and the contributions of $s$-channel $\Delta L=2$ scatterings. The resulting rate equation can be written in the formb

$$
\begin{aligned}
& \frac{s H_{N}}{z} \frac{\mathrm{d} Y_{\Delta \ell}}{\mathrm{d} z}=\left(1+\frac{Y_{N_{1}}}{Y_{N_{1}}^{\mathrm{eq}}}\right) \sum_{\alpha} \delta \gamma_{\ell_{\alpha} \phi}^{N_{1}}-\frac{1}{2} \sum_{\alpha} \frac{Y_{\Delta \ell_{\alpha}}}{Y_{\ell}^{\mathrm{eq}}} \gamma_{\ell_{\alpha} \phi}^{N_{1}} \\
& -2 \sum_{\alpha, \beta} \delta \gamma_{\bar{\ell}_{\beta} \phi}^{\prime \ell_{\alpha} \phi}-\sum_{\alpha, \beta} \frac{Y_{\Delta \ell_{\alpha}}}{Y_{\ell}^{\mathrm{eq}}} \gamma_{\bar{\ell}_{\beta} \phi}^{\prime \ell_{\alpha} \phi},
\end{aligned}
$$

where we have introduced the CP-odd rates $\delta \gamma_{\mathcal{Y}}^{\mathcal{X}} \equiv \gamma(\mathcal{X} \rightarrow \mathcal{Y})-\gamma(\overline{\mathcal{X}} \rightarrow \overline{\mathcal{Y}})$.

Looking more closely at the term proportional to the CP-odd decay rate $\delta \gamma_{\ell_{\alpha} \phi}^{N_{1}}$, it would appear that the asymmetry does not vanish, as it should, when the $\mathrm{RH}$ neutrinos are in equilibrium. It is for this reason that the $s$-channel $\Delta L=2$ scattering terms appear with a prime: we must only include those scattering terms that do not count processes already accounted for through the decay and inverse decay terms. This process is referred to as Real Intermediate State (RIS) subtraction 13.

In order to understand this procedure, it is convenient to consider the process $\ell_{\alpha} \phi \rightarrow \tilde{N} \rightarrow \bar{\ell}_{\beta} \bar{\phi}$ mediated by a heavy sneutrino $\tilde{N}$ (rather than a $\mathrm{RH}$ neutrino N) [14]. The squared matrix element for this process has the form

$$
\left|\mathcal{M}\left(\ell_{\alpha} \phi \rightarrow \bar{\ell}_{\beta} \bar{\phi}\right)\right|^{2}=\frac{\left|\mathcal{M}\left(\ell_{\alpha} \phi \rightarrow \tilde{N}\right)\right|^{2}\left|\mathcal{M}\left(\tilde{N} \rightarrow \bar{\ell}_{\beta} \bar{\phi}\right)\right|^{2}}{\left(p^{2}-M^{2}\right)^{2}+(M \Gamma)^{2}} .
$$

Making a pole-dominance approximation 14 for the squared modulus of the sneutrino propagator, the RIS contribution can be written

$\left|\mathcal{M}_{\mathrm{RIS}}\left(\ell_{\alpha} \phi \rightarrow \bar{\ell}_{\beta} \bar{\phi}\right)\right|^{2}=\frac{\pi}{M \Gamma} \theta\left(\sqrt{p^{2}}\right) \delta\left(p^{2}-M^{2}\right)\left|\mathcal{M}\left(\ell_{\alpha} \phi \rightarrow \tilde{N}\right)\right|^{2}\left|\mathcal{M}\left(\tilde{N} \rightarrow \bar{\ell}_{\beta} \bar{\phi}\right)\right|^{2}$

from which $\gamma_{\mathrm{RIS}}\left(\ell_{\alpha} \phi \rightarrow \bar{\ell}_{\beta} \bar{\phi}\right)=\gamma\left(\ell_{\alpha} \phi \rightarrow \bar{\ell}_{\beta} \bar{\phi}\right)-\gamma^{\prime}\left(\ell_{\alpha} \phi \rightarrow \bar{\ell}_{\beta} \bar{\phi}\right)$ can be obtained on thermal averaging. Although technically more involved, a completely equivalent result can be obtained for the exchange of a RH neutrino (see Ref. 14]). Putting everything together, it can then be shown (assuming only $N_{1}$ exchange) that 13,14

$$
\sum_{\beta} \delta \gamma_{\bar{\ell}_{\beta} \bar{\phi}}^{\prime \ell_{\alpha} \phi}=\delta \gamma_{\ell_{\alpha} \phi}^{N_{1}}+\mathcal{O}\left(\lambda^{4}\right)
$$

On substituting this into Eq. [9], we see that the scattering terms conspire to change the overall sign of the first term, such that the asymmetry vanishes in the equilibrium limit, as it should. Keeping only the (RIS corrected) decay and inverse decay terms, the equation for the asymmetry now reads

$$
\frac{s H_{N}}{z} \frac{\mathrm{d} Y_{\Delta \ell}}{\mathrm{d} z}=-\left(1-\frac{Y_{N_{1}}}{Y_{N_{1}}^{\mathrm{eq}}}\right) \sum_{\alpha} \delta \gamma_{\ell_{\alpha} \phi}^{N_{1}}-\frac{1}{2} \sum_{\alpha} \frac{Y_{\Delta \ell_{\alpha}}}{Y_{\ell}^{\mathrm{eq}}} \gamma_{\ell_{\alpha} \phi}^{N_{1}} .
$$

\footnotetext{
${ }^{\mathrm{b}}$ The rate equation can be recast in terms of $B-L$, the difference between the baryon and lepton number, which is conserved by the spectator processes. Here, we instead emphasize that we have dealt explicitly with only the doublet leptons and right-handed neutrinos. This notation is used later in Sec. 5.2
} 

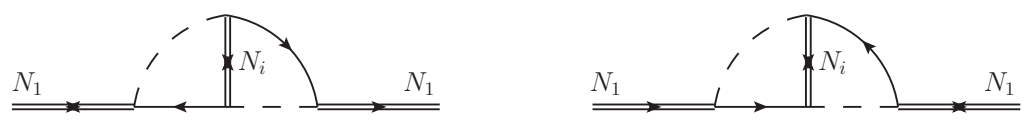

Fig. 1. Self-energy diagrams for the lightest Majorana neutrino, $N_{1}$ : direct contributions. Solid double lines stand for RH neutrinos, solid lines for SM lepton doublets and dashed lines for Higgs bosons. The neutrino propagator with forward arrow corresponds to $\left\langle 0\left|T\left(N_{k} \bar{N}_{k}\right)\right| 0\right\rangle$, whereas the neutrino propagators with forward-backward arrows correspond to $\left\langle 0\left|T\left(N_{k} N_{k}\right)\right| 0\right\rangle$ or $\left\langle 0\left|T\left(\bar{N}_{k} \bar{N}_{k}\right)\right| 0\right\rangle$.

The CP-odd rate $\delta \gamma_{\ell_{\alpha} \phi}^{N_{i}}$ can be written in terms of the CP-even rates $\gamma_{\ell_{\alpha} \phi}^{N_{i}}$ via $\delta \gamma_{\ell_{\alpha} \phi}^{N_{i}}=\epsilon_{i \alpha} \sum_{\beta} \gamma_{\ell_{\beta} \phi}^{N_{i}}$, where $\epsilon_{i \alpha}$ are the per-flavor CP asymmetries

$$
\epsilon_{i \alpha}=\frac{\Gamma\left(N_{i} \rightarrow \ell_{\alpha} \phi\right)-\Gamma\left(N_{i} \rightarrow \bar{\ell}_{\alpha} \bar{\phi}\right)}{\sum_{\beta}\left[\Gamma\left(N_{i} \rightarrow \ell_{\beta} \phi\right)+\Gamma\left(N_{i} \rightarrow \bar{\ell}_{\beta} \bar{\phi}\right)\right]} .
$$

For the $N_{1}$-dominated scenario and in the one-flavour approximation, the rate equations become (see, e.g., Refs. [4, 10])

$$
\begin{gathered}
\frac{\mathrm{d} Y_{N_{1}}}{\mathrm{~d} z}=-D_{1}\left(Y_{N_{1}}-Y_{N_{1}}^{\mathrm{eq}}\right), \\
\frac{\mathrm{d} Y_{\Delta \ell}}{\mathrm{d} z}=\epsilon_{1} D_{1}\left(Y_{N_{1}}-Y_{N_{1}}^{\mathrm{eq}}\right)-W Y_{\Delta \ell},
\end{gathered}
$$

where $\epsilon_{1} \equiv \sum_{\alpha} \epsilon_{1 \alpha}$. We can now identify the decay terms, proportional to

$$
D_{1} \equiv \frac{z}{H_{N} n_{N_{1}}^{\mathrm{eq}}} \sum_{\alpha} \gamma_{\ell_{\alpha} \phi}^{N_{1}}=K_{1} z \frac{\mathcal{K}_{1}(z)}{\mathcal{K}_{2}(z)}
$$

and the washout due to inverse decays, proportional to

$$
W \equiv \frac{1}{2} D_{1} \frac{Y_{N_{1}}^{\mathrm{eq}}}{Y_{\ell}^{\mathrm{eq}}}
$$

The factor $K_{1} \equiv \Gamma_{1} / H_{N}$, appearing in Eq. (17), is called the decay parameter. For the present scenario, $K_{1} \gg 1$, and we are in the so-called strong washout regime. In this case, $Y_{N_{1}} \approx Y_{N_{1}}^{\mathrm{eq}}$, and any initial asymmetry present before or generated during the production phase of the lightest RH neutrinos (at temperatures $T>M_{1}$ ) is completely washed out. We can then estimate the final asymmetry by setting the source and washout terms equal to one another 10,15 :

$$
Y_{\Delta \ell} \approx-\frac{\epsilon_{1}}{W} \frac{\mathrm{d} Y_{N_{1}}}{\mathrm{~d} z} \approx-\frac{\epsilon_{1}}{W} \frac{\mathrm{d} Y_{N_{1}}^{\mathrm{eq}}}{\mathrm{d} z}=\frac{2}{z K_{1}} \epsilon_{1} Y_{\ell}^{\mathrm{eq}},
$$

where $Y_{\ell}^{\text {eq }}=15 /\left(4 \pi^{2} g_{*}\right)$. We see that the final asymmetry is proportional to the $\mathrm{CP}$-violating parameter and inversely proportional to the decay factor $K$; if the $\mathrm{CP}$-violating parameter is too small or the washout too strong, the final asymmetry is suppressed.

At tree level, we have $\Gamma\left(N_{i} \rightarrow \ell_{\alpha} \phi\right)=\Gamma\left(N_{i} \rightarrow \bar{\ell}_{\alpha} \bar{\phi}\right)$, and the CP-violating parameter is zero. The sources of $\mathrm{CP}$ violation instead arise through the interference 

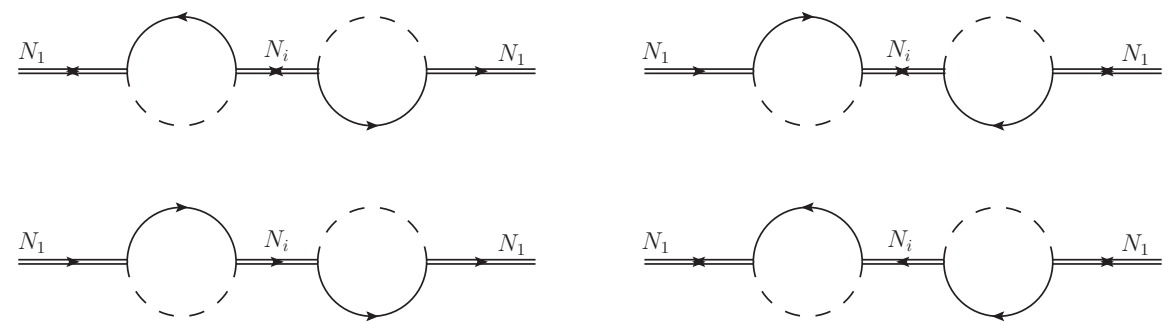

Fig. 2. Self-energy diagrams for the lightest Majorana neutrino, $N_{1}$ : indirect contributions.

of the tree-level and one-loop processes, pictured in Fig. 1 and Fig. 2 , The perflavour CP-violating parameters are then given by $[1,16$

$$
\begin{aligned}
\epsilon_{i \alpha}= & \frac{1}{8 \pi} \sum_{j \neq i} \frac{\operatorname{Im}\left[\lambda_{\alpha i}^{*}\left(\lambda^{\dagger} \lambda\right)_{i j} \lambda_{\alpha j}\right]}{\left|\lambda_{i}\right|^{2}} \xi\left(0, \frac{M_{j}^{2}}{M_{i}^{2}}\right) \\
& +\frac{1}{8 \pi} \sum_{j \neq i} \frac{\operatorname{Im}\left\{\lambda_{\alpha i}^{*}\left[\left(\lambda^{\dagger} \lambda\right)_{i j} M_{j}+M_{i}\left(\lambda^{\dagger} \lambda\right)_{j i}\right] \lambda_{\alpha j}\right\}}{\left|\lambda_{i}\right|^{2}} \frac{M_{i}}{M_{i}^{2}-M_{j}^{2}},
\end{aligned}
$$

where

$$
\xi(b, x)=\sqrt{x}\left[1+\frac{b}{1-x}-(1+x) \ln \frac{1+x}{x}\right]
$$

and the mass splittings of the $\mathrm{RH}$ neutrinos are assumed to be large compared to their characteristic decay width.

The first term on the right-hand side of Eq. 20 corresponds to the so-called direct or $\varepsilon^{\prime}$-type $\mathrm{CP}$ violation, which arises from the interference between the treelevel diagram and the one-loop vertex correction (see Fig. 1). The terms on the second line of Eq. 20 correspond to the so-called indirect or $\varepsilon$-type CP violation, which arises from the interference between the tree-level diagrams and the one-loop self-energy corrections (see Fig. 2). For mass splittings that are small compared to the characteristic decay width, the $\varepsilon$-type $\mathrm{CP}$ violation is enhanced and can dominate over the $\varepsilon^{\prime}$-type CP violation, leading to so-called resonant leptogenesis (see, e.g., Ref. [14] and chapters [11] and [17 of this review). Conversely, when the mass splittings are comparable to the masses themselves, the $\varepsilon$ - and $\varepsilon^{\prime}$-type $\mathrm{CP}$ violation are of similar magnitude. In the one-flavour regime, the lattermost term on the second line of Eq. 20) (proportional to $M_{i}^{2}$ ) does not contribute to the asymmetry, and we have

$$
\epsilon_{i}=\sum_{\alpha} \epsilon_{i \alpha}=\frac{1}{8 \pi} \sum_{j \neq i} \frac{\operatorname{Im}\left[\left(\lambda^{\dagger} \lambda\right)_{i j}^{2}\right]}{\left|\lambda_{i}\right|^{2}} \xi\left(1, \frac{M_{j}^{2}}{M_{i}^{2}}\right) .
$$

The remainder of this chapter will focus on the thermal field theoretic derivations of the key ingredients that we have introduced in this section. In so doing, we will see how the current state-of-the-art goes beyond the simplistic analysis and approximations that we have detailed above. 


\section{The right-handed neutrino production rate}

The purpose of this section is to consider in-vacuum and thermal corrections to the RH neutrino production rate in the simplest realization of thermal leptogenesis, described in Sec. 2. The neutrino production rate can be understood as the spacetime average of the rate at which the thermal plasma creates quanta of the lightest $\mathrm{RH}$ neutrino. In thermal equilibrium, the creation rate equals the destruction rate. Even though we are interested in out-of-equilibrium dynamics to address the lepton asymmetry generation, the $\mathrm{RH}$ neutrino production rate can be extracted in terms of an equilibrium distribution function. We label the production rate as $\gamma_{N_{1}} \equiv \gamma$, and we express it as the imaginary part of a retarded self-energy, $\Pi_{R}$, as follows 18,19

$$
\gamma=2 \int \frac{d^{3} \boldsymbol{k}}{(2 \pi)^{3}} \frac{\operatorname{Im} \Pi_{R}(\omega)}{\omega} f_{N}^{\mathrm{eq}}(\omega)
$$

where $\omega=\sqrt{k^{2}+M^{2}}$ with $k=|\boldsymbol{k}|, f_{N}^{\text {eq }}$ is the Fermi-Dirac distribution, the factor of two is due to the spin polarization. Moreover a neutrino width can be defined as

$$
\Gamma(\omega) \equiv \frac{\operatorname{Im}_{R}(\omega)}{\omega},
$$

that, at leading order in the couplings, at zero temperature and in the neutrino rest frame, simply reads $\Gamma_{\mathrm{LO}}^{T=0}=M|\lambda|^{2} /(8 \pi)$. The neutrino width is a more fundamental object than the neutrino production rate, see Eq. (23), and we are going to review its radiative and thermal corrections in what follows.

\subsection{In-vacuum and thermal corrections in the non-relativistic regime}

In the simplest realization of thermal leptogenesis, $M$ is much larger than the electroweak scale and then a great simplification can be obtained by taking all SM masses to be negligible with respect to the heavy-neutrino mass C However, some processes contributing to $\Gamma$ are infrared divergent in this limit. For example, the contribution coming from $2 \rightarrow 2$ scatterings (such as $A N \rightarrow \ell \phi$, where $A$ is any SM vector) are not infrared finite. The corresponding expressions are lengthy because some sort of regulator, e.g. thermal masses, has to be introduced for the infrared divergences 20,21 . Only summing all contributions at a given order in perturbation theory leads to a finite result 18 . For example, the process $A N \rightarrow \ell \phi$ has to be considered together with 3 -body decays, such as $N \rightarrow \ell \phi A$, and virtual corrections in order to obtain a finite result.

The relevant SM couplings involved in the next-to-leading order (NLO) calculation of $\Gamma$ are the $\mathrm{SU}(2)_{L}$ and $\mathrm{U}(1)_{Y}$ gauge couplings $g$ and $g^{\prime}$ respectively, the top Yukawa coupling $\lambda_{t}=\sqrt{2} m_{t} / v$ and the Higgs self interaction $\lambda_{h}=m_{h}^{2} /\left(2 v^{2}\right)$,

\footnotetext{
$\overline{{ }^{c} A t ~} T \gtrsim 160 \mathrm{GeV}$ the Higgs mechanism is not operative, hence SM particles can only get thermal masses of order $g T$. Nevertheless, the latter would be much smaller than the RH neutrino mass scale, $M \gg T \gg g T$.
} 


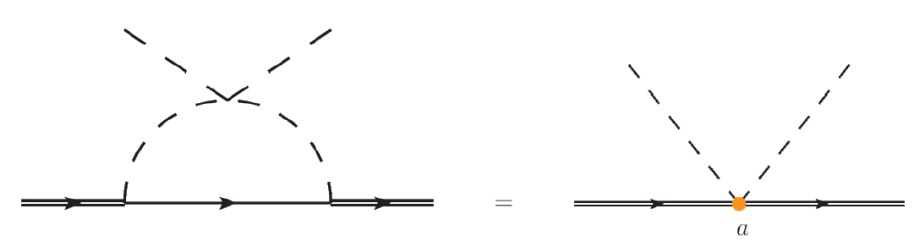

Fig. 3. One-loop matching condition for the neutrino-Higgs coupling. Double lines are RH neutrino propagators, single lines lepton propagators and dashed lines Higgs propagators.

where $m_{h}$ is the zero temperature Higgs mass. The NLO expression for $\Gamma$ in the rest frame of $N$ is 18,19

$$
\Gamma=\frac{M|\lambda|^{2}}{8 \pi}[1+\underbrace{\frac{29}{128 \pi^{2}}\left(3 g^{2}+g^{\prime 2}\right)}_{3 \%(2.5 \%)}-\underbrace{\frac{21 \lambda_{t}^{2}}{32 \pi^{2}}}_{5 \%(2 \%)}-\lambda_{h} \frac{T^{2}}{M^{2}}] .
$$

Only the leading thermal correction is displayed at this stage. Also, the numerical values for couplings defined in the $\overline{\mathrm{MS}}$ scheme and renormalized at the weak scale (at $10^{10} \mathrm{GeV}$ ) are shown, having fixed $\lambda_{h}=m_{h}^{2} /\left(2 v^{2}\right)$ and assumed $m_{h}=125 \mathrm{GeV}$.

The first three terms in Eq. (25) are the zero-temperature part of the width and radiative corrections are included that correspond to virtual corrections to $1 \rightarrow 2$ decays of $N$ as well as real corrections (i.e. $2 \rightarrow 2$ scatterings, $1 \rightarrow 3$ decays). They can be obtained with zero-temperature QFT methods. The temperature correction in Eq. 25) can be obtained by using the real-time formalism of thermal field theory. In the limit where $\lambda$ is small with respect to the relevant SM couplings, one can compute $\Gamma$ from the imaginary part of the $N$-propagator in the thermal plasma, computed by explicitly summing all possible "cuts" of the relevant Feynman diagrams. The finite-temperature cuts can be obtained by the so-called KobesSemenoff rules, which yield the absorptive parts of Feynman diagrams [22], and generalize the cutting rules valid at $T=0$.

The neutrino width has recently been recast in terms of a non-relativistic EFT for heavy Majorana fermions that may be dubbed Heavy Majorana Effective Theory (HMET) in analogy with the Heavy Quark Effective Theory 23. Indeed one may implement, at the Lagrangian level, the separation of the energy scales, $M \gg T$. The advantages of an EFT treatment for heavy particles can be summarized as follows. Firstly the EFT makes manifest, already at the Lagrangian level, the nonrelativistic nature of the Majorana particle. Secondly it allows to separate the computation of radiative and thermal corrections: radiative corrections are computed setting $T=0$ and contribute to the Wilson coefficients of the EFT, whereas thermal corrections are computed in the EFT as small corrections affecting the propagation of the non-relativistic RH neutrinos in the thermal medium. Finally, the power counting of the EFT allows a rather transparent organization of the calculation, leading to several simplifications that would not be obvious at the level of the relativistic thermal field theory. 


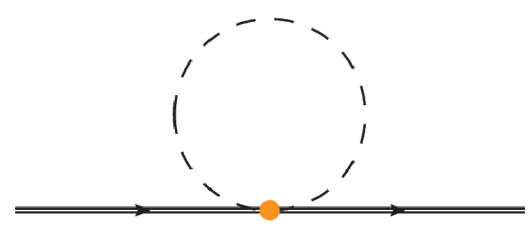

Fig. 4. Higgs tadpole contribution to the neutrino thermal width.

At an energy scale much smaller than $M$, the low-energy modes of the Majorana neutrino can be described by a field $\psi$, whose effective interactions with the SM particles are encoded in the EFT 23]

$$
\mathcal{L}_{\mathrm{EFT}}=\mathcal{L}_{\mathrm{SM}}+\mathcal{L}_{\psi}=\mathcal{L}_{\mathrm{SM}}+\bar{\psi}\left(i v \cdot \partial+\frac{i \Gamma^{T=0}}{2}\right) \psi+\frac{\mathcal{L}^{(1)}}{M}+\frac{\mathcal{L}^{(2)}}{M^{2}}+\frac{\mathcal{L}^{(3)}}{M^{3}}+\ldots
$$

The power counting of the EFT indicates that the leading operators responsible for the neutrino thermal decay are dimension-five operators contributing to $\mathcal{L}^{(1)}$. The symmetries of the EFT allow for only one possible dimension-five operator, which reads

$$
\mathcal{L}^{(1)}=a \bar{\psi} \psi \phi^{\dagger} \phi
$$

This operator describes the scattering of Majorana neutrinos with Higgs particles. By dimensional arguments, its contribution to a thermal width has to scale as $T^{2} / M$. The Wilson coefficient $a$ is fixed at one loop by the matching condition shown in Fig. 3. The left-hand side stands for an (in-vacuum) diagram in the fundamental theory in Eq. (1), whereas the right-hand side for an (in-vacuum) diagram in the EFT in Eq. (26). For the decay width, only the imaginary part of the matching coefficient is relevant; one finds $\operatorname{Im} a=-3|\lambda|^{2} \lambda_{h} /(8 \pi)$.

The thermal width induced by Eq. 27) can be computed from the tadpole diagram shown in Fig. 4, where the dashed line has to be understood now as a thermal Higgs propagator. The leading thermal width reads $18,19,23$,

$$
\Gamma_{\phi}^{T}=2 \frac{\operatorname{Im} a}{M}\left\langle\phi^{\dagger}(0) \phi(0)\right\rangle_{T}=-\frac{|\lambda|^{2} M}{8 \pi} \lambda_{h}\left(\frac{T}{M}\right)^{2},
$$

where $\left\langle\phi^{\dagger}(0) \phi(0)\right\rangle_{T}$ stands for the thermal condensate of the field $\phi$. The above expression of $\Gamma_{\phi}^{T}$ coincides with the thermal part of Eq. 25. Note that in the EFT the calculation has split into a one-loop matching, shown in Fig. 3, which can be done in vacuum, and the calculation of a one-loop tadpole diagram, shown in Fig. 4 , which is done in thermal field theory. In a similar fashion, one can calculate $T / M$ suppressed corrections to the thermal decay width. Only dimension-seven operators contribute to the width at next order in $T / M$, and they are included in $\mathcal{L}^{(3)}$. They describe couplings of the Majorana neutrino to Higgs bosons, leptons, quarks and gauge bosons respectively. Finally, the thermal width at first order in the SM couplings and at order $(T / M)^{4}$ (and in the neutrino rest frame) reads 19,23

$$
\Gamma^{T}=\frac{|\lambda|^{2} M}{8 \pi}\left[-\lambda_{h}\left(\frac{T}{M}\right)^{2}-\frac{\pi^{2}}{80}\left(3 g^{2}+g^{\prime 2}\right)\left(\frac{T}{M}\right)^{4}-\frac{7 \pi^{2}}{60}\left|\lambda_{t}\right|^{2}\left(\frac{T}{M}\right)^{4}\right]
$$


Corrections due to the $\mathrm{RH}$ neutrino motion are included to the above accuracy in Ref. [23 within the EFT framework, whereas they are fully taken into account in Ref. [19], together with zero-temperature quantum corrections at leading order in the SM couplings.

\subsection{Right-handed neutrino production rate in the relativistic regime}

After summing over helicities (denoted below by $s= \pm$ ) and including lepton chemical potentials (denoted by $\mu_{\alpha}$ ), the production rate of the $j$-th $\mathrm{RH}$ neutrino with momentum $k$ can be written as (a sum over active lepton flavor is understood)

$$
\begin{aligned}
\sum_{s} \dot{f}_{j}(k) & =\left|\lambda_{\alpha j}\right|^{2}\left\{\frac{\Gamma_{\alpha j}^{+}}{e^{\left(\omega_{j}-\mu_{\alpha}\right) / T}+1}+\frac{\Gamma_{\alpha j}^{-}}{e^{\left(\omega_{j}+\mu_{\alpha}\right) / T}+1}\right\}+O\left(\lambda^{4}\right), \\
\Gamma_{\alpha j}^{ \pm} & \equiv \frac{1}{2 \omega_{j}} \operatorname{Tr}\left[K_{j} \rho_{\alpha}\left( \pm K_{j}\right)\right] .
\end{aligned}
$$

Here $K_{j}=\left(\omega_{j}, \boldsymbol{k}\right), \omega_{j} \equiv \sqrt{k^{2}+M_{j}^{2}}$, and

$$
\rho_{\alpha}\left(K_{j}\right) \equiv \int_{-\infty}^{\infty} \mathrm{d} t \int_{\boldsymbol{x}} e^{i\left(\omega_{j} t-\boldsymbol{k} \cdot \boldsymbol{x}\right)}\left\langle\left\{\left(\phi^{c \dagger} \ell_{\alpha}\right)(t, \boldsymbol{x}),\left(\bar{\ell}_{\alpha} \phi^{c}\right)(0)\right\}\right\rangle
$$

is the so-called spectral function (twice the imaginary part of a retarded correlatord ${ }^{\mathrm{d}}$. The two contributions in Eq. (30) originate from leptons and antileptons, respectively. If the lepton chemical potentials vanish, i.e. $\mu_{\alpha}=0$, then $\Gamma_{\alpha j}^{+}=\Gamma_{\alpha j}^{-}$. The production rate is a physically relevant concept as long as the distribution function $f_{j}$ remains much below its equilibrium value, given by the Fermi-Dirac distribution. However, the same coefficients $\Gamma_{\alpha j}^{ \pm}$also govern the behavior of $f_{j}$ close to equilibrium [25], even though the form of the differential Eq. 30 is then different.

The task now is to compute $\Gamma_{\alpha j}^{ \pm}$within a SM plasma at a temperature $T$. The system has at least three different scales that affect the computation. One is the SM crossover temperature $T_{c} \sim 160 \mathrm{GeV}$ [26, 27], below which the Higgs mechanism is operative. The other two are the temperature $T$ and the Majorana mass $M_{j}$. It is important to note that even if we were only interested in leptogenesis, then the Higgs phase still needs to be considered, given that sphaleron processes, which partially convert lepton asymmetries into baryon asymmetries, only switch off at $T \sim 130 \mathrm{GeV}$ 28].

The techniques used and the precision of the computations that has been reached to date depend on the parametric regime considered, i.e. the relations of the three scales mentioned above. In the present section, we focus on the relativistic and ultrarelativistic regimes. The relativistic regime corresponds to $M_{j} \sim \pi T$, and the ultrarelativistic to $M_{j} \lesssim g T$, where $g$ denotes the weak gauge coupling. For $M_{j} \sim$

dThere exist different conventions for the spectral function, see, e.g. 24], where a factor $1 / 2$ appears in Eq. 32. 
$\mathrm{GeV}$ the ultrarelativistic regime covers temperatures $T \gtrsim 5 \mathrm{GeV}$, i.e. all temperatures of interest for leptogenesis. On the other hand, for $M_{j} \gtrsim \mathrm{TeV}$, both the relativistic and ultrarelativistic regimes are relevant at the early stages of the evolution, whereas the very late stages may be addressed with non-relativistic methods (see Sec. 3.1).

Starting with the relativistic regime, the main computational challenge is posed by the fact that when two scales are of similar magnitudes, $M_{j} \sim \pi T$, then no kinematic simplifications are possible, and both scales need to be retained. At NLO, this implies that computations become technically complicated [29]. Nevertheless all phase-space integrals appearing can be reduced into 2-dimensional ones [30], which can subsequently be evaluated numerically. The full NLO computation for this regime, assuming $T \gg 160 \mathrm{GeV}$, is described in Ref. [31], and it generalizes the non-relativistic result discussed in Sec. 3.1 to a broader temperature range.

If the temperature is increased so that $\pi T \gg M_{j}$, the relativistic results themselves break down. In this ultrarelativistic regime, infinite "resummations" (two nested resummations, Hard Thermal Loop resummation and Landau-PomeranchukMigdal resummation) are needed in order to obtain even the correct leading-order result. For $T>160 \mathrm{GeV}$, the techniques and results relevant for the ultrarelativistic regime were worked out in Refs. 32,34 . They have been extended to $T \lesssim 160 \mathrm{GeV}$ in Ref. 35. Also, a consistent way to interpolate between the ultrarelativistic and relativistic regimes has been worked out for $T>160 \mathrm{GeV}[36$, so that, for high temperatures, results applicable to any $M_{j}$ and $\pi T$ are available. Up-to-date numerical results for the rates in the various parametric regimes can be found through the web page http://www.laine.itp.unibe.ch/production-midT/

One price to pay for the resummations of the ultrarelativistic regime is that only the leading order in SM couplings has been reached so far. However, in principle, the NLO level can also be attacked; corrections could indeed be large, because they are only suppressed by $O(g)$ in this regime. In the context of particle production rates in a QCD plasma, the corresponding techniques have been worked out in Refs. 37, 38.

The above computations referred to a system in which chemical potentials had been set to zero. At $T \gtrsim 160 \mathrm{GeV}$, a non-zero lepton chemical potential was included in Refs. 39,40 . In addition, at low temperatures $T \lesssim 1 \mathrm{GeV}$, chemical potentials play a decisive role [41], and have been included in Refs. [42, 43].

Recently, it has been realized that the two helicity states of a massive RH neutrino, denoted by $s$ and summed together in Eq. (30), actually behave quite differently. This may lead to important physical effects. Rates for the specific helicity states were considered at $T<130 \mathrm{GeV}$ in Ref. [44] and at $T>130 \mathrm{GeV}$ in Ref. [40]. A partial earlier investigation at low temperatures can be found in Ref. [45], albeit for a toy model with a Dirac rather than a Majorana $\mathrm{RH}$ neutrino.

Apart from particle production, the same coefficients $\Gamma_{\alpha j}^{ \pm}$also play a role for lepton number washout rates [46], which represent an important ingredient in any 
leptogenesis computation. In this case, the coefficients $\Gamma_{\alpha j}^{ \pm}$come in combination with so-called lepton number susceptibilities, which have been computed up to NLO 46] and NNLO 47] at $T>160 \mathrm{GeV}$. At $T \sim 160 \mathrm{GeV}$ the susceptibilities suffer from infrared divergences related to light Higgs modes and would require a nonperturbative determination. Leading-order numerical results for the susceptibilities at $T<130 \mathrm{GeV}$ and $T>130 \mathrm{GeV}$ can be found on the web page http://www. laine.itp.unibe.ch/production-midT/, A recent discussion about the influence of susceptibilities at $T \sim 130 \mathrm{GeV}$ can be found in Ref. [48].

\section{CP-violating parameter from right-handed neutrino decays}

As discussed in Sec. 2. CP-violating parameters are a crucial ingredient entering the rate equations for the lepton-number asymmetry. They are related to the CP asymmetries generated by the Majorana neutrinos when decaying into leptons and antileptons carrying a flavor $\alpha$. We discuss thermal corrections to the CP asymmetry in the non-relativistic regime and then its connection with the lepton asymmetry.

\subsection{Towards NLO corrections to the CP-violating parameter in the non-relativistic regime}

At zero temperature and at zeroth order in the SM couplings, the RH neutrino CP asymmetries have been known for some time [10, 16. They may be computed from the diagrams shown in Fig. 1 and Fig. 2 by cutting through lepton and Higgs-boson lines.

Contributions from the diagrams of Fig. 1 are sometimes called direct contributions, because they are not resonant in the limit of nearly degenerate neutrinos. For the lightest Majorana neutrino, conventionally taken to be of type 1, they give (from Eq. 20p and Eq. (21))

$$
\epsilon_{1 \alpha}^{T=0, \text { direct }}=\frac{M_{i}}{M_{1}}\left[1-\left(1+\frac{M_{i}^{2}}{M_{1}^{2}}\right) \ln \left(1+\frac{M_{1}^{2}}{M_{i}^{2}}\right)\right] \frac{\operatorname{Im}\left[\left(\lambda_{1}^{*} \lambda_{i}\right)\left(\lambda_{\alpha 1}^{*} \lambda_{\alpha i}\right)\right]}{8 \pi\left|\lambda_{1}\right|^{2}} .
$$

Here and in the rest of this section, the neutrino-type index $i$ is understood as summed over all neutrino species different from $1(i>1)$.

Contributions from the diagrams of Fig. 2 are sometimes called indirect contributions, because they are resonant in the limit of nearly degenerate neutrinos (see Ref. 17] for more details). For the lightest RH neutrino, they give (again from Eq. (20))

$$
\epsilon_{1 \alpha}^{T=0, \text { indirect }}=\frac{M_{1} M_{i}}{M_{1}^{2}-M_{i}^{2}} \frac{\operatorname{Im}\left[\left(\lambda_{1}^{*} \lambda_{i}\right)\left(\lambda_{\alpha 1}^{*} \lambda_{\alpha i}\right)\right]}{8 \pi\left|\lambda_{1}\right|^{2}}+\frac{M_{1}^{2}}{M_{1}^{2}-M_{i}^{2}} \frac{\operatorname{Im}\left[\left(\lambda_{1} \lambda_{i}^{*}\right)\left(\lambda_{\alpha 1}^{*} \lambda_{\alpha i}\right)\right]}{8 \pi\left|\lambda_{1}\right|^{2}} .
$$

The distinction between direct and indirect contributions loses significance far away from the degenerate limit.

In a thermal medium, decay widths and CP asymmetry parameters appearing in the rate equations get thermal corrections. While the $\mathrm{RH}$ neutrino thermal 
decay width is known at first order in the SM couplings (see Sec. 3.2), this is not yet the case for the thermal corrections to the $\mathrm{RH}$ neutrino $\mathrm{CP}$ asymmetries generated by decays into leptons and antileptons, for which only partial results can be found in the literature 21,49 . Recently, however, the $\mathrm{RH}$ neutrino $\mathrm{CP}$ asymmetries have been computed in an expansion in $T / M_{k}$ and at first order in the SM couplings. The derivation is based on the HMET which makes the $1 / M_{k}$ expansion explicit at the Lagrangian level and allows an efficient organization of the calculation. The results may be useful if implemented in non-relativistic rate equations [50] for the late-time (low-temperature) evolution of the lepton-number asymmetry and, once a fully relativistic result valid for all temperatures is available, as a non-trivial constraint in the low-temperature regime. The results obtained in the HMET hold for temperatures lower than the lightest Majorana neutrino mass and (in the setting of Ref. 23) larger than the electroweak crossover scale. Including NLO contributions in the CP parameters, more general final states can appear in the heavy-neutrino decays, so that we define

$$
\epsilon_{k \alpha}=\frac{\Gamma\left(N_{k} \rightarrow \ell_{\alpha}+X\right)-\Gamma\left(N_{k} \rightarrow \bar{\ell}_{\alpha}+X\right)}{\sum_{\beta} \Gamma\left(N_{k} \rightarrow \ell_{\beta}+X\right)+\Gamma\left(N_{k} \rightarrow \bar{\ell}_{\beta}+X\right)},
$$

where $\Gamma$ is the width of the specified decay process and $X$ represents any other SM particle not carrying a lepton number.

We first review the hierarchical case, where the lightest neutrino mass $M_{1}$ is much smaller than the other neutrino masses $M_{i}$, with $i>1$ [51]. In this case, the full NLO result for the in-vacuum radiative corrections to the $\mathrm{CP}$-violating rates has been derived recently in Ref. 52. Thermal corrections to the CP asymmetry of the lightest neutrino were computed in terms of an expansion in the Yukawa couplings, SM couplings, $\left(M_{1} / M_{i}\right)$ and $\left(T / M_{1}\right)$; they read

$$
\begin{array}{r}
\epsilon_{1 \alpha}^{T}=-\frac{3}{16 \pi} \frac{M_{1}}{M_{i}} \frac{\operatorname{Im}\left[\left(\lambda_{1}^{*} \lambda_{i}\right)\left(\lambda_{\alpha 1}^{*} \lambda_{\alpha i}\right)\right]}{\left|\lambda_{1}\right|^{2}}\left[\left(-\frac{5}{3} \lambda_{h}+\frac{2 g^{2}+g^{\prime 2}}{12}\right)\left(\frac{T}{M_{1}}\right)^{2}\right. \\
\left.+\frac{7 \pi^{2}}{20}\left|\lambda_{t}\right|^{2}\left(\frac{T}{M_{1}}\right)^{4}+\left(\frac{5}{6} \lambda_{h}-\frac{2 g^{2}+g^{\prime 2}}{24}\right) \frac{k^{2} T^{2}}{M_{1}^{4}}\right] .
\end{array}
$$

This expression is accurate at fourth order in the Yukawa couplings, at order $M_{1} / M_{i}$ and at first order in the SM couplings, and, for each coupling, it provides the leading thermal correction. It also provides the leading thermal correction proportional to the three-momentum of $N_{1}$.

The leading thermal corrections proportional to the Higgs self-coupling $\lambda_{h}$ and to the gauge couplings $g$ and $g^{\prime}$ are of relative order $\left(T / M_{1}\right)^{2}$, whereas those proportional to the top Yukawa coupling $\left|h_{t}\right|^{2}$ are of relative order $\left(T / M_{1}\right)^{4}$. We show the different contributions in Fig. 5. At low temperatures, thermal corrections proportional to the Higgs self-coupling and to the gauge couplings dominate, whereas at temperatures closer to the neutrino mass, the suppression in $T / M_{1}$ becomes less important and the most numerically relevant corrections turn out to be those 


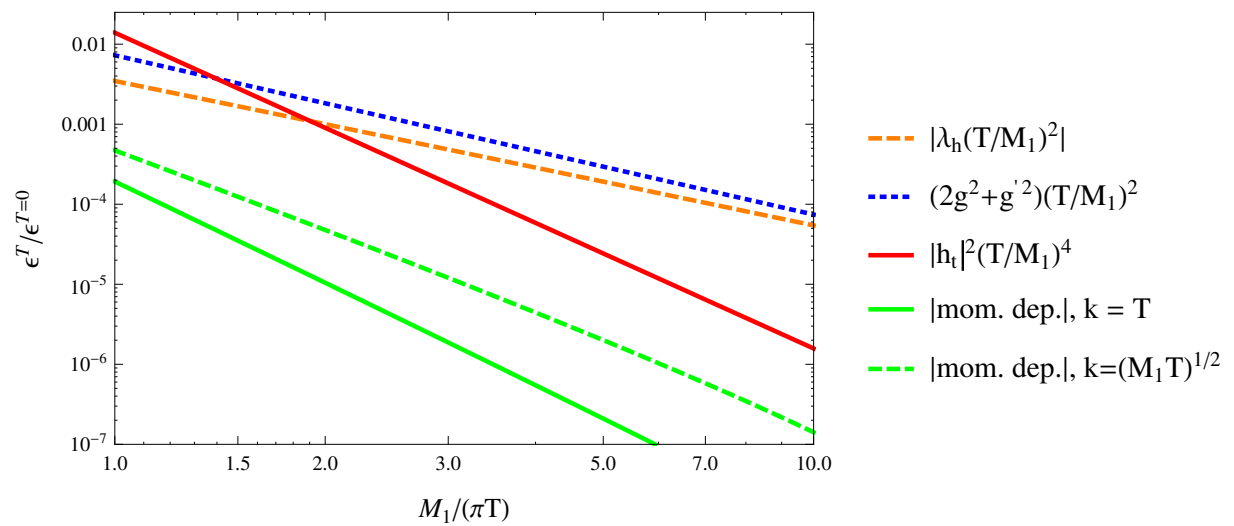

Fig. 5. Thermal corrections to the $\mathrm{CP}$ asymmetry of a $\mathrm{RH}$ neutrino decaying into leptons and antileptons as a function of the temperature in the hierarchical case 51]. The orange dashed line shows the contribution proportional to the Higgs self-coupling (the sign of the contribution has been changed to make it positive), the blue dotted line shows the contribution proportional to the gauge couplings and the red continuous line shows the contribution proportional to the top Yukawa coupling. The green lines show the leading thermal contribution proportional to the neutrino momentum (also in this case the sign of the contribution has been changed to make it positive). For the green continuous line, we take the neutrino momentum to be $T$, whereas, for the green dashed line, we take it to be $\sqrt{M_{1} T}$. The SM couplings have been computed at the scale $\pi T$ with one-loop running. The different thermal contributions to the $\mathrm{CP}$ asymmetry have been normalized with respect to the zero-temperature result expanded at leading order in $M_{1} / M_{i}$. The neutrino mass has been taken $M_{1}=10^{7} \mathrm{GeV}$.

proportional to the top Yukawa coupling. In Fig. 5 we also show the thermal contribution to the $\mathrm{CP}$ asymmetry due to a moving Majorana neutrino, which is of relative order $k^{2} T^{2} / M_{1}^{4}$. We plot this contribution for the case of a neutrino with momentum $T$ and for the case of a neutrino in thermal equilibrium with momentum $\sqrt{M_{1} T}$. We see that, for the considered momenta, the effect of a moving neutrino on the thermal CP asymmetry is tiny. In general, thermal effects are small in the hierarchical case, being at most of the order of a few percent for temperatures around the lightest neutrino mass. CP asymmetry and thermal effects may get enhanced if the two lightest neutrinos have almost degenerate masses.

Thermal corrections to the $\mathrm{CP}$ asymmetry in the case of two almost degenerate RH neutrinos with masses $M_{1}$ and $M_{2}$, and $0<\Delta=M_{2}-M_{1} \ll M_{1}$, were computed in Ref. 53. In the nearly degenerate case, it makes sense to distinguish between direct and indirect contributions. It is because of the indirect contributions, which can be resonant, that the $\mathrm{CP}$ asymmetry and its thermal corrections may become enhanced in the nearly degenerate case. The thermal corrections to the 
direct CP asymmetries for the two RH neutrinos $N_{1}$ and $N_{2}$ at rest read

$$
\begin{aligned}
\epsilon_{1 \alpha}^{T, \text { direct }}= & \frac{\operatorname{Im}\left[\left(\lambda_{1}^{*} \lambda_{2}\right)\left(\lambda_{\alpha 1}^{*} \lambda_{\alpha 2}\right)\right]}{8 \pi\left|\lambda_{1}\right|^{2}}\left(\frac{T}{M_{1}}\right)^{2}\left\{\lambda_{h}\left[2-\ln 2+(1-3 \ln 2) \frac{\Delta}{M_{1}}\right]\right. \\
& \left.-\frac{g^{2}}{16}\left[2-\ln 2+(3-5 \ln 2) \frac{\Delta}{M_{1}}\right]-\frac{g^{\prime 2}}{48}\left[4-\ln 2+(1-5 \ln 2) \frac{\Delta}{M_{1}}\right]\right\} \\
\epsilon_{2 \alpha}^{T, \text { direct }}= & -\frac{\operatorname{Im}\left[\left(\lambda_{1}^{*} \lambda_{2}\right)\left(\lambda_{\alpha 1}^{*} \lambda_{\alpha 2}\right)\right]}{8 \pi\left|\lambda_{2}\right|^{2}}\left(\frac{T}{M_{1}}\right)^{2}\left\{\lambda_{h}\left[2-\ln 2-(9-5 \ln 2) \frac{\Delta}{M_{1}}\right]\right. \\
& \left.-\frac{g^{2}}{16}\left[2-\ln 2-7(1-\ln 2) \frac{\Delta}{M_{1}}\right]-\frac{g^{\prime 2}}{48}\left[4-\ln 2-(9-7 \ln 2) \frac{\Delta}{M_{1}}\right]\right\} \\
& +\frac{\operatorname{Im}\left[\left(\lambda_{2}^{*} \lambda_{1}\right)\left(\lambda_{\alpha 1}^{*} \lambda_{\alpha 2}\right)\right]}{2 \pi\left|\lambda_{2}\right|^{2}}\left(\frac{T}{M_{1}}\right)^{2} \lambda_{h} \frac{\Delta}{M_{1}},
\end{aligned}
$$

which are accurate at fourth-order in the Yukawa couplings, at first order in the SM couplings, at order $\Delta / M_{1}$ and at order $\left(T / M_{1}\right)^{2}$. The leading thermal corrections to the indirect $\mathrm{CP}$ asymmetries for the two Majorana neutrinos at rest read

$$
\begin{aligned}
& \epsilon_{1 \alpha}^{T, \text { indirect }}=-\frac{\epsilon_{1 \alpha}^{T=0, \text { indirect }}}{3}\left(\left|\lambda_{2}\right|^{2}-\left|\lambda_{1}\right|^{2}\right) \frac{M_{1}}{\Delta} \frac{T^{2}}{M_{1}^{2}}, \\
& \epsilon_{2 \alpha}^{T, \text { indirect }}=-\frac{\epsilon_{2 \alpha}^{T=0, \text { indirect }}}{3}\left(\left|\lambda_{2}\right|^{2}-\left|\lambda_{1}\right|^{2}\right) \frac{M_{1}}{\Delta} \frac{T^{2}}{M_{1}^{2}},
\end{aligned}
$$

which are accurate at zeroth-order in the SM couplings and at leading order in $\Delta / M_{1}$. The $T=0 \mathrm{CP}$ asymmetry $\epsilon_{1 \alpha}^{T=0 \text {,indirect }}$ may be read from Eq. 34 for $i=2$, whereas $\epsilon_{2 \alpha}^{T=0 \text {,indirect }}$ follows from $\epsilon_{1 \alpha}^{T=0 \text {,indirect }}$ after the change $1 \leftrightarrow 2$.

The Yukawa coupling combination $\operatorname{Im}\left[\left(\lambda^{\dagger} \lambda\right)_{21}\left(\lambda_{\alpha 1}^{*} \lambda_{\alpha 2}\right)\right]$ in Eq. 38 is absent in the expression of the direct CP asymmetry for the neutrino of type 1 in Eq. (37). The origin of this contribution can be traced back to the kinematically allowed transition $N_{2} \rightarrow N_{1}$ when $M_{2}>M_{1}$, which provides an additional source of CP asymmetry.

For what concerns the indirect CP asymmetry, we see that both the $T=0$ contribution in Eq. (34) and the thermal contributions in Eq. (39) and Eq. (40) become large for $\Delta$ close to zero, i.e. in the nearly degenerate limit. For mass differences $\Delta$ comparable with the widths $\Gamma_{k}$ of the Majorana neutrinos, these should be resummed in the neutrino propagators. The resummation of the widths amounts to the replacement

$$
\frac{1}{\Delta} \rightarrow \frac{\Delta}{\Delta^{2}+\left(\Gamma_{2}-\Gamma_{1}\right)^{2} / 4},
$$

in the $T=0$ and thermal expressions of the CP asymmetries (explicit expressions can be found in Ref. [53]). At leading order, the RH-neutrino widths stem from the decay into a lepton and a Higgs boson and are $\Gamma_{k} \approx M_{k}\left|\lambda_{k}\right|^{2} /(8 \pi)$. To capture the saturation of the resonant enhancement for $\Delta \lesssim \Gamma_{k}$, the replacement in Eq. (41) is not sufficient. Instead, it is necessary to include also coherent transitions between the Majorana neutrino states, as described in detail in chapter [17] of this review. 

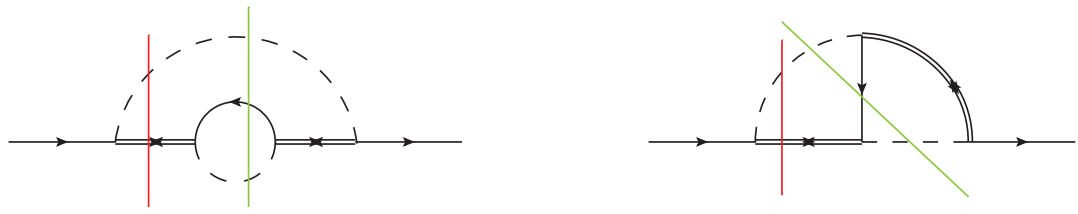

Fig. 6. Two-loop contributions to the lepton self-energy at $\mathcal{O}\left(\lambda^{4}\right)$. The red and green lines illustrate cuts that correspond to decay (red) and scattering (green) contributions within the Boltzmann approach.

\subsection{CP-violating parameter and lepton asymmetry with non- equilibrium quantum field theory}

Within non-equilibrium field theory, an equation of motion for the lepton asymmetry can be obtained starting from the lepton density, which is related to the temporal component of the lepton number current

$$
n_{L}=\frac{1}{V} \int_{V} d^{3} \boldsymbol{x}\left\langle J^{0}(t, \boldsymbol{x})\right\rangle, \quad J^{\mu} \equiv \sum_{\alpha=e, \mu, \tau} \bar{\ell}_{\alpha}(x) \gamma^{\mu} \ell_{\alpha}(x) .
$$

Its time evolution, using the Schwinger-Dyson equation for the lepton two-point function $S_{\alpha \beta}(x, y)=\left\langle T_{\mathcal{C}} \ell_{\alpha}(x) \bar{\ell}_{\beta}(y)\right\rangle$, is given by 5459

$$
\left(\partial_{t}+3 H\right) n_{L}=-\frac{1}{V} \int d^{3} \boldsymbol{x} \int_{\mathcal{C}} d^{4} y \operatorname{tr}\left[\Sigma_{\alpha \beta}(x, y) S_{\beta \alpha}(y, x)-S_{\alpha \beta}(x, y) \Sigma_{\beta \alpha}(y, x)\right] .
$$

Here, $x_{0}=t, T_{\mathcal{C}}$ indicates time-ordering along the closed-time path $\mathcal{C}$, and $y^{0}$ is integrated over $\mathcal{C}, \Sigma_{\beta \alpha}$ is the lepton self-energy. For a nearly CP-symmetric state, the right-hand side can be expanded in the lepton chemical potential, $\partial_{t} n_{L}=$ $S-W+\ldots$, where the zeroth-order contribution is the source term $S$ and the first order is the washout term $W$. The leading source term can be obtained by inserting the two-loop lepton self-energy at order $\lambda^{4}$, shown in Fig. 6, and expanding all propagators around a CP-symmetric state [59]. Furthermore, when using a Kadanoff-Baym ansatz for all propagators [60], and assuming that only $N_{1}$ deviates from equilibrium and that $S_{\alpha \beta} \propto \delta_{\alpha \beta}$ (unflavored regime), one obtains for times $t \gg 1 / M_{1}, 1 / T$ 57, 58, 60, 61 and up to terms $\mathcal{O}\left(e^{-M_{i} / T}\right.$ ) (see Ref. 62 for a discussion of these contributions)

$$
S=4\left|\lambda_{1}\right|^{2} \int_{k p q}(2 \pi)^{4} \delta(p-k-q) p \cdot k \epsilon_{1}(p, k, q) \delta f_{N \boldsymbol{p}}(t)\left(1-f_{\ell}^{\mathrm{eq}}(k)+f_{\phi}^{\mathrm{eq}}(q)\right),
$$

where $\delta f_{N \boldsymbol{p}}(t)=f_{N}(p, t)-f_{N}^{\mathrm{eq}}(p)$ denotes the deviation of the $N_{1}$-distribution from thermal equilibrium, $\int_{k}=\int \frac{d^{3} k}{2 \omega_{k}(2 \pi)^{3}}, f_{\ell}^{\mathrm{eq}}(k)=1 /\left(e^{\beta \omega_{k}}+1\right), f_{\phi}^{\mathrm{eq}}(q)=1 /\left(e^{\beta \omega_{q}}-1\right)$, and

$$
\epsilon_{1}(p, k, q)=\frac{\operatorname{Im}\left[\left(\lambda_{1}^{*} \lambda_{i}\right)^{2}\right]}{8 \pi\left(\lambda^{\dagger} \lambda\right)_{11}}\left(\frac{M_{1} M_{i}}{M_{1}^{2}-M_{i}^{2}} \frac{k^{\mu} L_{\mu}(p)}{p \cdot k}-\frac{1}{2} \frac{M_{1}}{M_{i}} \frac{k^{\mu} K_{\mu i}(p, q)}{p \cdot k}\right),
$$


where

$$
\begin{aligned}
L_{\mu}(p) & \equiv 16 \pi \int_{k^{\prime} q^{\prime}}(2 \pi)^{4} \delta\left(p-k^{\prime}-q^{\prime}\right) k_{\mu}^{\prime}\left(1-f_{\ell}^{\mathrm{eq}}\left(k^{\prime}\right)+f_{\phi}^{\mathrm{eq}}\left(q^{\prime}\right)\right), \\
K_{\mu i}(p, q) & \equiv 16 \pi \int_{k^{\prime} q^{\prime}}(2 \pi)^{4} \delta\left(p-k^{\prime}-q^{\prime}\right) k_{\mu}^{\prime}\left(1-f_{\ell}^{\mathrm{eq}}\left(k^{\prime}\right)+f_{\phi}^{\mathrm{eq}}\left(q^{\prime}\right)\right) \frac{M_{i}^{2}}{M_{i}^{2}-\left(q-k^{\prime}\right)^{2}} .
\end{aligned}
$$

Let us stress that the unflavored CP asymmetry in Eq. 45 comprises thermal effects, which are included through the thermal distribution functions in $L_{\mu}(p)$ and $K_{\mu i}(p, q)$. The two terms in $\epsilon_{1}$ arise from the two diagrams in Fig. 6] and correspond to wave- and vertex-contributions, respectively. At finite temperature, the integral $L_{\mu}$ can be expressed as $[57,60]$

$$
\begin{aligned}
L_{0} & =\frac{2 T}{y} I_{1}\left(y_{0}, y\right), \\
L_{i} & =\frac{2 p_{i}}{y^{3}}\left(y_{0} I_{1}\left(y_{0}, y\right)-\frac{1}{2}\left(y_{0}^{2}-y^{2}\right)\left(1+x_{\ell}-x_{\phi}\right) I_{0}\left(y_{0}, y\right)\right),
\end{aligned}
$$

where $y_{0}=p_{0} / T, y=|p| / T, x_{\ell(\phi)}=m_{\ell(\phi)} / T$, and

$$
I_{n}\left(y_{0}, y\right) \equiv \int_{z_{-}}^{z_{+}} d z z^{n}\left(1+\frac{1}{e^{y_{0}-z}-1}-\frac{1}{e^{z}+1}\right)
$$

with $z_{ \pm}=\left[y_{0}\left(1+x_{\ell}-x_{\phi}\right) \pm y \lambda^{\frac{1}{2}}\left(1, x_{\ell}, x_{\phi}\right)\right] / 2$. For $T, m_{\ell}, m_{\phi} \rightarrow 0$, the integrals are given by $L_{\mu}(p) \rightarrow p_{\mu}$ and $K_{\mu i}(p, q) \rightarrow-2 p_{\mu} x_{i}\left(1-\left(1+x_{i}\right) \ln \left(1+1 / x_{i}\right)\right)$, where $x_{i}=M_{i}^{2} / p^{2}$ and $p^{2}=M_{1}^{2}$. For $M_{i} \gg M_{1}$, both integrals agree, i.e. $K_{\mu i}(p, q) \rightarrow$ $L_{\mu}(p)$. Explicit analytic results for $I_{n}$ in the massless limit $m_{\ell}=m_{\phi}=0$ can be found in Ref. [57. In the zero-temperature limit, the CP-violating parameter approaches the usual vacuum expression, i.e. when adding Eq. (33) and Eq. (34) and summing over the lepton flavor $\alpha$. In this limit, the source term in Eq. (44) agrees with the conventional Boltzmann result (cf. Sec. 2). At finite temperature, the source term in Eq. (44) can equivalently be written in the form

$$
\begin{aligned}
S & =8 \operatorname{Im}\left[\left(\lambda_{1}^{*} \lambda_{i}\right)^{2}\right] \int_{p k q k^{\prime} q^{\prime}}(2 \pi)^{4} \delta(p-k-q)(2 \pi)^{4} \delta\left(p-k^{\prime}-q^{\prime}\right) k^{\mu} k_{\mu}^{\prime} M_{1} M_{i} \delta f_{N \boldsymbol{p}}(t) \\
& \times\left(\frac{1}{M_{1}^{2}-M_{i}^{2}}-\frac{1}{2} \frac{1}{M_{i}^{2}-\left(q-k^{\prime}\right)^{2}}\right)\left(1-f_{\ell}^{\mathrm{eq}}(k)+f_{\phi}^{\mathrm{eq}}(q)\right)\left(1-f_{\ell}^{\mathrm{eq}}\left(k^{\prime}\right)+f_{\phi}^{\mathrm{eq}}\left(q^{\prime}\right)\right) .
\end{aligned}
$$

This form allows for a direct comparison to the Boltzmann result: the first factor $\left(1-f_{\ell}^{\mathrm{eq}}(k)+f_{\phi}^{\mathrm{eq}}(q)\right)$ originates from the quantum statistical terms in the Boltzmann equation, while the second factor $\left(1-f_{\ell}^{\mathrm{eq}}\left(k^{\prime}\right)+f_{\phi}^{\mathrm{eq}}\left(q^{\prime}\right)\right)$ would be absent in the usual Boltzmann treatment and takes into account thermal corrections to the CP-violating loop amplitude. A notable property is that the source term is actually symmetric with respect to the interchange $q, k \leftrightarrow q^{\prime}, k^{\prime}$ (using that $q-k^{\prime}=q^{\prime}-k$ ). This can be understood within the closed-time path approach: the source term originates from closing the lepton self-energies shown in Fig. 6, with the external lines connected by 
a lepton propagator. In this diagram, both lepton/Higgs lines with momenta $q, k$ and $q^{\prime}, k^{\prime}$, respectively, appear symmetrically巳

Furthermore, within the Boltzmann approach, it is necessary to rely on the socalled RIS subtraction procedure to obtain a consistent source term (see Sec. 2), which can be cumbersome when including quantum-statistical terms and thermal effects $21,60,64$. This is not the case within the closed-time path formalism, where consistency comes "for free". The correspondence between the closed-time path formalism and the Boltzmann approach can be illustrated by considering all possible cuts of the self-energies, which are indicated by the colored lines in Fig. 6 .

The source term can be further simplified in the strongly hierarchical limit $M_{j} \gg$ $M_{1}$ :

$$
S=\frac{\left|\lambda_{1}\right|^{2}}{4 \pi} \epsilon_{1}^{T=0} \int_{p} L^{\mu}(p) L_{\mu}(p) \delta f_{N \boldsymbol{p}}(t) .
$$

Quantitatively, including the finite-temperature corrections leads to an enhancement of the CP asymmetry that is exponentially suppressed for $T \ll M_{1}$ and numerically of $\mathcal{O}(1)$ for $T \sim M_{1}$. When naively taking into account thermal masses for the lepton and Higgs, the enhancement of the CP asymmetry becomes smaller again for even larger temperatures. When $T \sim M_{1} / g$, the source term discussed above vanishes, and one expects a resummation similar to the one described for $N_{1}$-production to become necessary to capture the leading-order result.

\section{Different approaches to rate equations for particle dynamics in the early Universe}

In this section, the evolution equations for leptogenesis are addressed. These equations are needed to obtain the time evolution of RH neutrino and lepton-asymmetry number densities. First, we discuss how the Boltzmann equations (BEs) can be derived from a full quantum mechanical set of equations and different methods to solve the latter. Afterwards, we move to effective kinetic equations, valid to all orders in the SM interactions and obtained by the main assumption of a large separation between the time scales of leptogenesis. NLO corrections to the rates are easier to handle in this second approach.

\subsection{On the applicability and limitation of standard Boltzmann equations}

As discussed in Sec. 2, the traditional approach to understanding the kinematics of leptogenesis is based on BEs. The underlying physical picture is one of classical particles that propagate freely between occasional scattering processes. It is however clear that this approach has to be generalized and improved: a net lepton number generation is a pure quantum phenomenon, which takes place in the hot

\footnotetext{
e This can lead to cancellations in scenarios where the CP asymmetry is a purely thermal effect, for example in the context of soft leptogenesis 63 .
} 
early Universe. Moreover, the Boltzmann equations suffer from the so-called double counting problem, which can be solved only in some limiting cases. These issues have motivated more careful analyses in recent years: how are particles with finite lifetime properly accommodated in the Boltzmann picture, which assumes asymptotic initial and final states? Can the (quasi-)particles be defined consistently in a medium at finite density? Is the quantum interference that makes the difference between decays into particles and antiparticles affected by the medium?

Questions like these need to be addressed on a case-by-case basis for the various conceivable leptogenesis scenarios. To this end, the derivation has to start from a consistent set of quantum equations whose applicability is not in question. For the late-time limit of hierarchical thermal leptogenesis, these considerations have led to slight modifications $[57 \sqrt[62]{6}$ but otherwise put the Boltzmann approach on a sounder basis. Since, without strong assumptions, the proper quantum equations are hard to solve directly, there is also a great interest in obtaining generalized quantum kinetic equations simple enough for phenomenological analyses. These may be of a Boltzmann-like form, even if the physical picture of freely propagating quasiparticles is not applicable (i.e. Boltzmann-type equations obtained under different or weaker assumptions).

A suitable starting point are self-consistent Schwinger-Dyson equations or an equivalent set of Kadanoff-Baym equations (KBEs) 65], which are full equations of motion for out-of-equilibrium quantum systems. They are normally obtained via the Schwinger-Keldysh formalism 66. The basic objects under study are the expectation values of the two-point functions of the fields with the time argument belonging to the closed-time path. As far as leptogenesis is concerned, Higgs bosons, leptons/antileptons and Majorana neutrinos are the most important. Let us call the generic two-point function $G(x, y)$, which obeys a Schwinger-Dyson equation $G^{-1}(x, y)=G_{0}^{-1}(x, y)-\Pi(x, y)$, where the free two-point function $G_{0}$ and selfenergy $\Pi$ enter. KBEs are integro-differential equations that relate the physically relevant quantities of the out-of-equilibrium quantum fields with the imaginary and real parts of the retarded and advanced self-energies of the system $f$ If the selfenergies are obtained in a consistent manner (2PI effective action/Phi-derivable approximation), KBEs are known to be a suitable starting point for the derivation of conserving quantum kinetic equations. It is useful to express the two-point function $G(x, y)$ in terms of the spectral function and statistical function, $G^{-}(x, y)$ and $G^{+}(x, y)$ respectively 8 An example of a KBE is written in Eq. 43 in Sec. 4.2

These equations are hard to assess directly but may be solved through different sets of approximations. If one insists on obtaining an evolution equation for a one-particle distribution function, its definition in terms of the quantum objects of

\footnotetext{
${ }_{\mathrm{f}}$ In out-of-equilibrium dynamics, the Kubo-Martin-Schwinger relation is not satisfied so the system cannot be described by just one propagator. A stringent derivation of BEs will necessarily involve a definition that relates those quantum objects to distribution functions or number densities. ${ }^{\mathrm{g}}$ The definition of the spectral and statistical correlator is $G^{-}(x, y) \equiv i\langle[\phi(x), \phi(y)]\rangle$ and $G^{+}(x, y) \equiv\langle\{\phi(x), \phi(y)\}\rangle / 2$ for a scalar field, see e.g. 67.
} 
the KBEs is one of several necessary steps. In typical leptogenesis scenarios, the uncertainties are mostly in the solution of the KBEs for the RH neutrinos, whose evolution can depart far from equilibrium and whose mixing can be relevant for the generation of the asymmetry. Since the quantitatively-controlled first-principles derivation is key to answering the questions stated above, we sketch two known paths that lead to Boltzmann-type equations in the following. A complete analysis will usually include numerical estimates of the accuracy of the various approximations made.

First, we discuss the Fourier-space method, which relies on the separation of the slow macroscopic evolution of mean coordinates and the fast microscopic evolution 60, 68 70. It requires the transformation to the Wigner space coordinates $(x, y) \rightarrow$ $(X, s)$, where $\mathbf{s}=\mathbf{x}-\mathbf{y}, \Delta t=x_{0}-y_{0}, \mathbf{X}=(\mathbf{x}+\mathbf{y}) / 2, t=\left(x_{0}+y_{0}\right) / 2$, where $\mathbf{s}$ and $\Delta t$ are related to the microscopic scales $\left(\Delta t \sim 1 / M_{k}\right)$, and $\mathbf{X}$ and $t$ to the macroscopic scales $\left(t \sim 1 / \Gamma_{k}, 1 / H\right)$. Consequently a Fourier transform is performed in the microscopic variables $\mathbf{s}$ and $\Delta t$, which give the momentum of the out-of-equilibrium field $\mathbf{k}$ and a frequency $\omega$, which can be related to the energy at later times. The soobtained equations are still exact but not useful in practice. The next step consists of a gradient expansion in $t$ by exploiting the small changes in the mean coordinates. If the system is sufficiently close to equilibrium, one has for the relevant contributions $\Delta t \ll t$ and, since a homogeneous and isotropic Universe is assumed, one may drop $\mathrm{X}$. The solutions obtained for the spectral function $G^{-}(x, y)$ incorporate both the thermal masses and a thermal width, traced back to the real and imaginary parts of the self-energies entering the corresponding evolution equation. Keeping only first-order contributions, quantum-kinetic equations that generalize the Boltzmann equations can be obtained for the statistical propagator.

One may progress further in this direction by assuming a proportional relationship between the statistical propagator and the spectral function that generalizes their equilibrium relationship (Kadanoff-Baym ansatz). Using an approximate quasi-particle solution for the spectral function, which is proportional to $\delta\left(p^{2}-m^{2}\right)$, puts the momentum on the mass shell. This latter step allows the integration of the energy coordinate of the four-momenta. Its accuracy should be checked by comparing with finite-width results. If multiple on-shell peaks happen to be close-by and overlap, such as in resonant leptogenesis, it is not necessarily good (see the companion chapter [17]). Otherwise it defines one-particle distribution functions and the Boltzmann-type equations that govern their evolution.

A second approach makes use of the Wentzel-Kramers-Brillouin (WKB) approximation 71 73. In contrast to the gradient expansion, the WKB method does not rely on a Fourier transform in relative times (also known as the "two-time formalism" 67]). It also works far out-of-equilibrium and does not rely on an on-shell approximation or any other a priori assumption about the form of the correlation functions, such as the Kadanoff-Baym ansatz. The statistical propagator can be expressed in terms of a generalized distribution function that follows a generalized 
$\mathrm{BE}$, i.e. a first-order differential equation that is local in time. The accuracy of the $\mathrm{BE}$ is controlled by the accuracy of the WKB solution compared to the full KBE. The solutions found with this method are valid under the very general physical assumptions of weak coupling and separation of macroscopic and microscopic time scales.

The smallness of the parameters that control the accuracy of the WKB solution for $G^{ \pm}$is also required for the convergence of the gradient expansion in the Wignerspace approach. Hence, the applicability of both techniques is similar. The latter has a closer relationship to the diagrammatic S-matrix expansion in vacuum as both are performed in momentum space. If the finite width is taken into account, a full resummation is, however, required. On the other hand, the WKB method does not depend on such a resummation and treats this issue in a more intuitive manner.

\subsection{Effective kinetic equations and real-time correlation functions at finite temperature}

There are several circumstances that make leptogenesis relatively simple and allow for a relatively rigorous theoretical treatment: (i) most degrees of freedom are in thermal equilibrium, because their equilibration rate is much larger than the Hubble expansion rate $H$; (ii) there is a separation of time scales: the degrees of freedom which are out of equilibrium evolve much more slowly than most other degrees of freedom and (iii) the system is homogeneous in space. The Hubble rate is typically of the same order as, or larger than the equilibration rate of the slowly changing, or slow for short, degrees of freedom. Therefore, the fast degrees of freedom create a quasi-equilibrium state, which is characterized by the temperature $T$ and the values of the slow ones.

In the absence of expansion the time derivative of the slow degrees of freedom $y_{a}$ can only depend on the temperature and on the values of the $y_{a}$. We choose the $y_{a}$ such that their expectation values vanish in complete thermal equilibrium. The typical time scale on which they evolve is smaller than the inverse temperature. The non-equilibrium state of the system is then completely determined by the temperature, and the $y_{a}$ h If the $y_{a}$ are sufficiently small, one can expand to linear order, so that, in the absence of expansion, the effective kinetic equations take the form

$$
\dot{y}_{a}=-\gamma_{a b} y_{b}
$$

The real coefficients $\gamma_{a b}$ only depend on the temperature. One should stress that these equations do not require the validity of any Boltzmann equation or quasiparticle picture. The only approximation is based on the separation of time scales.

What the slow variables are depends on the details of the model parameters. As an example consider the simplest case of thermal leptogenesis where (i) the

${ }^{\mathrm{h}}$ It could also depend on the chemical potentials of practically conserved charges, but we assume that these vanish. 
masses of the RH neutrinos are hierarchical, $M_{1} \ll M_{i}$; (ii) the $N_{1}$-decay rate is much larger than $H$, which is the so-called strong washout regime; (iii) the rates for charged-lepton Yukawa interactions are much smaller than $H$; (iv) the weak sphaleron rate is much larger than $H$. The asymmetry is then generated when $T$ is smaller than $M_{1}$, and when there are only $N_{1}$ 's present in the plasma. Furthermore, the $\mathrm{RH}$ neutrinos are non-relativistic. To a first approximation, one can neglect their motion completely. Then the only slow variables are the $N_{1}$-number density $n_{N_{1}}$, and the baryon minus lepton number density $n_{B-L}$ Taking into account the Hubble expansion, the effective kinetic equations (51] become [50]

$$
\begin{aligned}
D_{t} n_{N_{1}} & =-\gamma_{N_{1}}\left(n_{N_{1}}-n_{N_{1}}^{\mathrm{eq}}\right)-\gamma_{N_{1}, B-L} n_{B-L}, \\
D_{t} n_{B-L} & =-\gamma_{B-L, N_{1}}\left(n_{N_{1}}-n_{N_{1}}^{\mathrm{eq}}\right)-\gamma_{B-L} n_{B-L},
\end{aligned}
$$

where $D_{t} \equiv d / d t+3 H$, and $n_{N_{1}}^{\text {eq }}$ is the equilibrium number density of $N_{1}$. Equation (52) and Eq. (53) are valid to all orders in SM couplings and on time scales much larger than the equilibration time of the fast degrees of freedom.

The coefficients $\gamma_{a b}$ in Eq. (51) can be determined in thermal field theory by using the theory of quasi-stationary fluctuations 74 . Even in thermal equilibrium, the $y_{a}$ are not constant in time but fluctuate around their equilibrium values. Their thermal fluctuations can be described by the same classical equations of motion as Eq. (51), except that there is an additional Gaussian white noise, which describes the effect of fluctuations of the fast variables. These equations can be used to compute the real-time autocorrelation functions of the fluctuations of $y_{a}$. These correlation functions can also be computed in the underlying microscopic quantum field theory. For $\omega \ll \omega_{\mathrm{UV}}$, where $\omega_{\mathrm{UV}}$ is the characteristic frequency of the spectator processes, the two results have to match. In this way, one obtains the relation

$$
\gamma_{a b}=T \omega \operatorname{Im} \Pi_{a c}^{R}(\omega)\left(\chi^{-1}\right)_{c b}, \quad\left(\gamma \ll \omega \ll \omega_{\mathrm{UV}}\right) .
$$

for the rates in Eq. 511) it is similar to the Kubo relations for transport coefficients, and it contains the retarded self-energy $\Pi_{a c}^{R}$ of $y_{a}$ and $y_{c}$, as well as the real and symmetric matrix of susceptibilities $\chi_{a b} \equiv\left\langle y_{a} y_{b}\right\rangle$.

Now one has to identify operators which represent the slowly changing variables in the microscopic quantum field theory. There are two different types of operators entering the susceptibilities and the retarded self-energies in Eq. 544: the ones containing SM fields, and the ones containing only the RH neutrinos. The SM fields are in kinetic equilibrium, and the corresponding operators are global charges which would be conserved if certain Yukawa interactions are neglected,

$$
X_{a}=\int d^{3} x \bar{\ell} \gamma_{0} T_{a}^{\ell} \ell+\text { contributions from other fermions. }
$$

\footnotetext{
${ }^{i}$ At very high $T$, one only has to account for a single lepton number, which is the one which is produced in the $N_{1}$ decays. At lower temperature, when charged-lepton Yukawa interactions are in equilibrium, one has to account for different lepton flavors.

${ }^{\mathrm{j}}$ Here, the relation $\gamma \ll \omega$ has to be understood parametrically. Please note that $\gamma$ need not be the neutrino production rate. Equation 54 can also be used for the washout rate and for the asymmetry rate (and it has been used for that).
} 
Here, $T_{a}^{\ell}$ is the generator of the corresponding symmetry transformation acting on the left-handed lepton doublets $\ell_{\alpha}$. The RH neutrinos interact only very weakly. Without any interaction, the occupation number $f_{\boldsymbol{k}}$ of each field mode with momentum $\boldsymbol{k}$ would be conserved. Assuming a homogeneous Universe, we do not need any variables describing spatial variations. For a free field, the spin-averaged occupation number in a finite volume $V$ is

$$
f_{\boldsymbol{k}}=\frac{1}{2} \sum_{s} c_{\boldsymbol{k} s}^{\dagger} c_{\boldsymbol{k} s}
$$

with creation and annihilation operators $c_{\boldsymbol{k} s}^{\dagger}$ and $c_{\boldsymbol{k} s}$. To include the Yukawa interaction of the RH neutrinos, as given in the Lagrangian (1), we use the interaction picture. Then the sterile-neutrino field operator has the same form as in the free case, and we define the occupation number in the interaction picture by Eq. (56) 75] If there are several flavors of RH neutrinos present in the plasma, one has to work with a matrix of occupation numbers (see also the accompanying chapter of this review [11]).

We expand the retarded two-point functions in Eq. (54) in the very small Yukawa couplings $\lambda$ and keep only the leading order. They can be then factored out and no longer know about the scale $\gamma$, so that the restriction $\omega \gg \gamma$ in the matching formula (54) can be dropped, and we can take the limit $\omega \rightarrow 0$. Furthermore, one can integrate out the $\mathrm{RH}$ neutrinos treating them as free fields. One is left with two- or four-point functions of composite operators made of SM fields. Consider, e.g., the washout rate $\gamma_{a b}$ in the effective kinetic equation

$$
\dot{X}_{a}=-\gamma_{a b} X_{b}+\cdots .
$$

If the small charged-lepton Yukawa interactions are neglected one finds 46

$$
\gamma_{a b}=\frac{1}{2} T V \sum_{i} \lambda_{\alpha i}^{*}\left\{T_{a}^{\ell}, T_{c}^{\ell}\right\}_{\alpha \beta} \lambda_{\beta i}\left(\chi^{-1}\right)_{c b} \mathcal{W}\left(M_{i}\right) .
$$

Here, one sees the factorized structure; the function

$$
\mathcal{W}\left(M_{i}\right) \equiv-\int \frac{d^{3} k}{(2 \pi)^{3}} \frac{f_{\text {eq }, N}^{\prime}\left(\omega_{i}\right)}{2 \omega_{i}} \operatorname{Tr}\{k[\rho(k)+\rho(-k)]\}_{k^{0}=\omega_{i}},
$$

with $\omega_{i} \equiv\left(k^{2}+M_{i}^{2}\right)^{1 / 2}$, does not depend on the sterile-neutrino Yukawa couplings $\lambda_{\alpha i}$. The prime on the Fermi-Dirac distribution for the RH neutrino denotes a derivative with respect to the energy and the fundamental object is a two-point function of the composite operators of SM fields

$$
\rho(k) \equiv \frac{1}{3} \int \mathrm{d}^{4} x e^{i k \cdot x}\left\langle\left\{\left(\phi^{c \dagger} \ell_{\alpha}\right)(x),\left(\bar{\ell}_{\alpha} \phi^{c}\right)(0)\right\}\right\rangle_{0} .
$$

The trace in Eq. (59) is over Dirac spinor indices, and the subscript 0 in Eq. (60) indicates that the thermal average is performed with $\lambda=0$. The factor $1 / 3$ is due to a flavor average.

\footnotetext{
kote that the product $V\left(\chi^{-1}\right)_{c b}$ is finite in the limit $V \rightarrow \infty$.
} 
The final step in computing the rates is to evaluate the correlation functions of the SM fields, as well as the susceptibilities. At leading order in $\lambda$, the susceptibilities of the sterile-neutrino occupation can be computed in the free-field limit. The susceptibilities of the charges in Eq. (55) have been computed in Refs. [46, 47] to quadratic order in the SM couplings. Interestingly, the leading correction to the free-field result is parametrically of linear order due to infrared effects.

Recently, the approach described in this section has also been applied to the asymmetry rate $\gamma_{B-L, N_{1}}$ 52]. It has been computed at NLO in SM couplings at $T=0$ in the hierarchical limit, and the corrections were found to be of order $5 \%$. Thus, thermal leptogenesis is theoretically well under control. Also recently, the approach just described, based on the separation of time scales and the resulting identification of the slow modes, has been applied to leptogenesis at $T \gtrsim M$ with $\mathrm{RH}$ neutrino masses below the electroweak scale [40]. A set of equations resembling the schematic structure of Eqs. 52 and 53 has been derived in the same spirit of this section, using a density matrix to keep track of the slow sterile-neutrino degrees of freedom, i.e. momentum, flavour and helicity, since it is found that the two helicity states are produced and equilibrate at different rates in the presence of a lepton asymmetry (see also Refs. 44, 45]).

\section{Conclusions and outlook}

If the baryon asymmetry in the Universe was generated via leptogenesis, the $\mathrm{RH}$ neutrinos experienced a thermal medium of SM particles. It is a non-trivial task to include thermal effects to the different ingredients of the heavy-neutrino dynamics: production rate, CP asymmetries and washout rates. Those quantities enter the evolution equations for the $\mathrm{RH}$ neutrino and lepton-asymmetry number densities. In order to include the effects of the SM heat bath systematically, NLO calculations at finite temperature are needed. Hence, thermal field theory is the tool to achieve a better knowledge of the key ingredients for leptogenesis.

The RH neutrino production rate is known at NLO (in-vacuum and thermal corrections) for all the kinematic regimes satisfying $M \gtrsim \pi T$. A broad temperature range is covered, namely temperatures larger and smaller than the SM electroweak crossover $T_{c} \sim 160 \mathrm{GeV}$. Different approaches have provided the same result for the non-relativistic regime, where NLO thermal corrections are power suppressed (see Eq. (29)).

The status of the $\mathrm{CP}$ asymmetries in $\mathrm{RH}$ neutrino decays is as follows. Thermal corrections at zeroth order in SM couplings are known and they are exponentially suppressed when $T \ll M$ (see Sec. 4.2), whereas they can be of numerical relevance for temperatures closer to the neutrino mass. The NLO in-vacuum result has been recently carried out for a hierarchical heavy-neutrino mass spectrum 52]. On the other hand, NLO thermal corrections have been studied in the framework of nonrelativistic EFTs. For the hierarchical case, the leading thermal corrections, of relative order $\left(T / M_{1}\right)^{2}$, are known, whereas the subleading ones, of relative order 
$\left(T / M_{1}\right)^{4}$, comprise the contribution from the top-Yukawa coupling only at the time of this review. For the nearly degenerate case, thermal corrections of relative order $\left(T / M_{1}\right)^{2}$ have been derived so far. Such results are obtained in an unbroken phase of the SM. Completing the thermal NLO result in the non-relativistic regime appears possible in the near future. Moreover, the derivation of the $\mathrm{CP}$ asymmetries in the relativistic regime is desirable, so as to cover a broader temperature range. A three-loop thermal calculation is what is needed to accomplish such a goal.

Lepton-number washout rates come as a combination of the neutrino width and lepton-number susceptibilities. The latter are known at NLO and NNLO at $T>160$ $\mathrm{GeV}$. At leading order, numerical results are also available for $T<160 \mathrm{GeV}$.

The Boltzmann equations are the traditional approach to address the numberdensity evolution of $\mathrm{RH}$ neutrinos and lepton asymmetry. They rely on several approximations that have to be tested case-by-case for different leptogenesis scenarios. We have reviewed recent developments that allow for a rigorous derivation of Boltzmann equations from more fundamental quantum kinetic equations. The latter are not easy to solve and therefore approximation methods are needed (see Sec.5.1. Moreover, we discussed a complementary approach that relies only on the separation of time scales appearing in leptogenesis (see Sec. 5.2). These effective kinetic equations are valid to all orders in the SM couplings. Indeed radiative corrections have already been successfully included in this framework. The neutrino production rate, $\mathrm{CP}$ asymmetries and washout rates are seen as the coefficients dictating the evolution of RH neutrino and lepton-asymmetry occupation numbers and they are traced back to correlation functions at finite temperature.

Finally, we comment on the different technical approaches discussed in this chapter. In Sec. 3.1, the imaginary part of the retarded self-energy in the real-time formalism is considered, $\Pi_{R}=\Pi_{11}+\Pi_{12}$, which is related to the neutrino thermal width. In the strict non-relativistic regime it suffices looking at the $\Pi_{11}$ component, since $\Pi_{12}$ is exponentially suppressed in the heavy-mass limit. Then the very same quantity is understood in terms of a spectral function in Sec. 3.1. whose relation to the retarded self-energy reads $\rho=2 \operatorname{Im} \Pi_{R}$ (actually all the correlation functions can be expressed in terms of $\rho$ in thermal equilibrium) and the imaginary-time formalism is adopted. There, one first computes the Euclidean self-energy, which is then analytically continued into the retarded self-energy. In Sec. 4.2 we introduce the lepton asymmetry in the closed-time path formalism. Here, the expectation values are taken along the original Schwinger-Keldysh contour as for the real-time formalism. This formulation of thermal field theory is known to be better suited to address directly out-of-equilibrium dynamics, since it corresponds to evolving the initial density operator in time along this contour. In the equilibrium case, the vertical part of the real-time contour is indeed the initial Boltzmann density operator. We further remark that the lepton asymmetry originates from the imaginary part of closed-time path self-energies. On the same footing, the imaginary part of correlation functions is the key ingredient for obtaining the coefficients of 
the evolution equations. As we saw in Sec. 5.2, one ends up relating the imaginary part of retarded self-energies via a fluctuation-dissipation argument to the washout rate. The method sketched there also includes the effect of the time evolution of the initial density operator, which in this case factors the equilibrated fast modes from the out-of-equilibrium slow modes. The time evolution is governed by the equations of motions for the operators describing the slow modes.

\section{Acknowledgements}

We gratefully acknowledge the hospitality of the Munich Institute for Astro- and Particle Physics (MIAPP) of the DFG cluster of excellence "Origin and Structure of the Universe", where this work has been initiated. The work of S.B. and M.L. was supported by the Swiss National Science Foundation (SNF) under grant 200020168988. N.B. and A.V. acknowledge support from the DFG cluster of excellence "Origin and structure of the Universe" (www.universe-cluster.de). The work of S.M. was supported by FONDECYT 11130118. The work of P.M. was supported by STFC Grant No. ST/L000393/1 and a Leverhulme Trust Research Leadership Award. P.M. would like to thank Pasquale Di Bari and Stéphane Lavignac for helpful discussions. A.S. acknowledges support from the grant 669668 - NEO-NAT - ERC-AdG-2014.

\section{References}

[1] M. Fukugita and T. Yanagida, Baryogenesis Without Grand Unification, Phys. Lett. B174, 45-47, (1986).

[2] M. Drewes, The Phenomenology of Right Handed Neutrinos, Int. J. Mod. Phys. E22, 1330019, (2013).

[3] V. A. Kuzmin, V. A. Rubakov, and M. E. Shaposhnikov, On the Anomalous Electroweak Baryon Number Nonconservation in the Early Universe, Phys. Lett. B155, 36, (1985).

[4] W. Buchmüller, P. Di Bari, and M. Plümacher, Leptogenesis for pedestrians, Annals Phys. 315, 305-351, (2005).

[5] S. Davidson, E. Nardi, and Y. Nir, Leptogenesis, Phys. Rept. 466, 105-177, (2008).

[6] W. Buchmüller and M. Plümacher, Baryon asymmetry and neutrino mixing, Phys. Lett. B389, 73-77, (1996).

[7] G. L. Fogli, E. Lisi, A. Marrone, A. Palazzo, and A. M. Rotunno, Evidence of $\theta_{13}>0$ from global neutrino data analysis, Phys. Rev. D84, 053007, (2011).

[8] S. Blanchet and P. Di Bari, The minimal scenario of leptogenesis, New J. Phys. 14, 125012, (2012)

[9] A. Pilaftsis, Heavy Majorana neutrinos and baryogenesis, Int. J. Mod. Phys. A14, 1811-1858, (1999).

[10] C. S. Fong, E. Nardi, and A. Riotto, Leptogenesis in the Universe, Adv. High Energy Phys. 2012, 158303, (2012).

[11] P. S. B. Dev, P. Di Bari, B. Garbrecht, S. Lavignac, P. Millington, and D. Teresi, Flavor effects in leptogenesis, arXiv:1711.02861, (2017).

[12] M. A. Luty, Baryogenesis via leptogenesis, Phys. Rev. D45, 455-465, (1992). 
[13] E. W. Kolb and S. Wolfram, Baryon Number Generation in the Early Universe, Nucl. Phys. B172, 224, (1980). [Erratum: Nucl. Phys.B195,542(1982)].

[14] A. Pilaftsis and T. E. J. Underwood, Resonant leptogenesis, Nucl. Phys. B692, 303-345, (2004).

[15] C. S. Fong and J. Racker, On fast CP violating interactions in leptogenesis, JCAP. 1007, 001, (2010).

[16] L. Covi, E. Roulet, and F. Vissani, CP violating decays in leptogenesis scenarios, Phys. Lett. B384, 169-174, (1996).

[17] P. S. B. Dev, M. Garny, J. Klaric, P. Millington, and D. Teresi, Resonant enhancement in leptogenesis, arXiv:1711.02863, (2017).

[18] A. Salvio, P. Lodone, and A. Strumia, Towards leptogenesis at NLO: the right-handed neutrino interaction rate, JHEP. 08, 116, (2011).

[19] M. Laine and Y. Schroder, Thermal right-handed neutrino production rate in the non-relativistic regime, JHEP. 02, 068, (2012).

[20] R. Barbieri, P. Creminelli, A. Strumia, and N. Tetradis, Baryogenesis through leptogenesis, Nucl. Phys. B575, 61-77, (2000).

[21] G. F. Giudice, A. Notari, M. Raidal, A. Riotto, and A. Strumia, Towards a complete theory of thermal leptogenesis in the SM and MSSM, Nucl. Phys. B685, 89-149, (2004).

[22] M. L. Bellac, Thermal field theory, Cambridge University Press. (1996).

[23] S. Biondini, N. Brambilla, M. A. Escobedo, and A. Vairo, An effective field theory for non-relativistic Majorana neutrinos, JHEP. 12, 028, (2013).

[24] M. Laine and A. Vuorinen, Basics of Thermal Field Theory, Lect. Notes Phys. 925, pp.1-281, (2016).

[25] D. Bödeker, M. Sangel, and M. Wormann, Equilibration, particle production, and self-energy, Phys. Rev. D93(4), 045028, (2016).

[26] M. Laine and M. Meyer, Standard Model thermodynamics across the electroweak crossover, JCAP. 1507(07), 035, (2015).

[27] M. D'Onofrio and K. Rummukainen, Standard model cross-over on the lattice, Phys. Rev. D93(2), 025003, (2016).

[28] M. D'Onofrio, K. Rummukainen, and A. Tranberg, Sphaleron Rate in the Minimal Standard Model, Phys. Rev. Lett. 113(14), 141602, (2014).

[29] B. Garbrecht, F. Glowna, and M. Herranen, Right-Handed Neutrino Production at Finite Temperature: Radiative Corrections, Soft and Collinear Divergences, JHEP. 04, 099, (2013).

[30] M. Laine, Thermal 2-loop master spectral function at finite momentum, JHEP. 05, 083, (2013).

[31] M. Laine, Thermal right-handed neutrino production rate in the relativistic regime, JHEP. 08, 138, (2013).

[32] D. Besak and D. Bödeker, Hard Thermal Loops for Soft or Collinear External Momenta, JHEP. 05, 007, (2010).

[33] A. Anisimov, D. Besak, and D. Bödeker, Thermal production of relativistic Majorana neutrinos: Strong enhancement by multiple soft scattering, JCAP. 1103, 042, (2011).

[34] D. Besak and D. Bödeker, Thermal production of ultrarelativistic right-handed neutrinos: Complete leading-order results, JCAP. 1203, 029, (2012).

[35] J. Ghiglieri and M. Laine, Neutrino dynamics below the electroweak crossover, JCAP. 1607(07), 015, (2016).

[36] I. Ghisoiu and M. Laine, Right-handed neutrino production rate at $T>160 \mathrm{GeV}$, JCAP. 1412(12), 032, (2014).

[37] J. Ghiglieri, J. Hong, A. Kurkela, E. Lu, G. D. Moore, and D. Teaney, Next-to-leading 
order thermal photon production in a weakly coupled quark-gluon plasma, JHEP. 05, 010, (2013)

[38] J. Ghiglieri and G. D. Moore, Low Mass Thermal Dilepton Production at NLO in a Weakly Coupled Quark-Gluon Plasma, JHEP. 12, 029, (2014).

[39] P. Hernandez, M. Kekic, J. Lopez-Pavon, J. Racker, and J. Salvado, Testable Baryogenesis in Seesaw Models, JHEP. 08, 157, (2016).

[40] J. Ghiglieri and M. Laine, GeV-scale hot sterile neutrino oscillations: a derivation of evolution equations, JHEP. 05, 132, (2017).

[41] X.-D. Shi and G. M. Fuller, A New dark matter candidate: Nonthermal sterile neutrinos, Phys. Rev. Lett. 82, 2832-2835, (1999).

[42] J. Ghiglieri and M. Laine, Improved determination of sterile neutrino dark matter spectrum, JHEP. 11, 171, (2015).

[43] T. Venumadhav, F.-Y. Cyr-Racine, K. N. Abazajian, and C. M. Hirata, Sterile neutrino dark matter: Weak interactions in the strong coupling epoch, Phys. Rev. D94 (4), 043515, (2016)

[44] S. Eijima and M. Shaposhnikov, Fermion number violating effects in low scale leptogenesis, Phys. Lett. B771, 288-296, (2017).

[45] L. Lello, D. Boyanovsky, and R. D. Pisarski, Production of heavy sterile neutrinos from vector boson decay at electroweak temperatures, Phys. Rev. D95(4), 043524, (2017).

[46] D. Bödeker and M. Laine, Kubo relations and radiative corrections for lepton number washout, JCAP. 1405, 041, (2014).

[47] D. Bödeker and M. Sangel, Order $g^{2}$ susceptibilities in the symmetric phase of the Standard Model, JCAP. 1504(04), 040, (2015).

[48] S. Eijima, M. Shaposhnikov, and I. Timiryasov, Freeze-out of baryon number in lowscale leptogenesis, arXiv:1709.07834, (2017).

[49] L. Covi, N. Rius, E. Roulet, and F. Vissani, Finite temperature effects on CP violating asymmetries, Phys. Rev. D57, 93-99, (1998).

[50] D. Bödeker and M. Wörmann, Non-relativistic leptogenesis, JCAP. 1402, 016, (2014).

[51] S. Biondini, N. Brambilla, and A. Vairo, CP asymmetry in heavy Majorana neutrino decays at finite temperature: the hierarchical case, JHEP. 09, 126, (2016).

[52] D. Bödeker and M. Sangel, Lepton asymmetry rate from quantum field theory: NLO in the hierarchical limit, JCAP. 1706(06), 052, (2017).

[53] S. Biondini, N. Brambilla, M. A. Escobedo, and A. Vairo, CP asymmetry in heavy Majorana neutrino decays at finite temperature: the nearly degenerate case, JHEP. 03, 191, (2016). [Erratum: JHEP08,072(2016)].

[54] W. Buchmüller and S. Fredenhagen, Quantum mechanics of baryogenesis, Phys. Lett. B483, 217-224, (2000).

[55] A. De Simone and A. Riotto, Quantum Boltzmann Equations and Leptogenesis, JCAP. 0708, 002, (2007).

[56] M. Garny, A. Hohenegger, A. Kartavtsev, and M. Lindner, Systematic approach to leptogenesis in nonequilibrium QFT: Vertex contribution to the CP-violating parameter, Phys. Rev. D80, 125027, (2009).

[57] M. Beneke, B. Garbrecht, M. Herranen, and P. Schwaller, Finite Number Density Corrections to Leptogenesis, Nucl. Phys. B838, 1-27, (2010).

[58] A. Anisimov, W. Buchmüller, M. Drewes, and S. Mendizabal, Quantum Leptogenesis I, Annals Phys. 326, 1998-2038, (2011). [Erratum: Annals Phys.338,376(2011)].

[59] M. Garny, A. Kartavtsev, and A. Hohenegger, Leptogenesis from first principles in the resonant regime, Annals Phys. 328, 26-63, (2013). 
[60] T. Frossard, M. Garny, A. Hohenegger, A. Kartavtsev, and D. Mitrouskas, Systematic approach to thermal leptogenesis, Phys. Rev. D87(8), 085009, (2013).

[61] M. Garny, A. Hohenegger, and A. Kartavtsev, Medium corrections to the CPviolating parameter in leptogenesis, Phys. Rev. D81, 085028, (2010).

[62] B. Garbrecht, Leptogenesis: The Other Cuts, Nucl. Phys. B847, 350-366, (2011).

[63] B. Garbrecht and M. J. Ramsey-Musolf, Cuts, Cancellations and the Closed Time Path: The Soft Leptogenesis Example, Nucl. Phys. B882, 145-170, (2014).

[64] P. S. Bhupal Dev, P. Millington, A. Pilaftsis, and D. Teresi, Flavour Covariant Transport Equations: an Application to Resonant Leptogenesis, Nucl. Phys. B886, 569664, (2014).

[65] G. Baym and L. P. Kadanoff, Conservation Laws and Correlation Functions, Phys. Rev. 124, 287-299, (1961).

[66] L. V. Keldysh, Diagram technique for nonequilibrium processes, Zh. Eksp. Teor. Fiz. 47, 1515-1527, (1964). [Sov. Phys. JETP20,1018(1965)].

[67] M. Drewes, S. Mendizabal, and C. Weniger, The Boltzmann Equation from Quantum Field Theory, Phys. Lett. B718, 1119-1124, (2013).

[68] J. Berges and M. M. Muller. Nonequilibrium quantum fields with large fluctuations. In 285th Heraeus Seminar: Interdisciplinary Workshop on Progress in Nonequilibrium Greens Functions (Kadanoff-Baym Equations II) Dresden, Gremany, August 19-23, 2002, (2002). URL http://alice.cern.ch/format/showfull?sysnb=2337540.

[69] T. Prokopec, M. G. Schmidt, and S. Weinstock, Transport equations for chiral fermions to order h bar and electroweak baryogenesis. Part 1, Annals Phys. 314, 208-265, (2004).

[70] M. Garny, A. Hohenegger, and A. Kartavtsev, Quantum corrections to leptogenesis from the gradient expansion, arXiv:1005.5385, (2010).

[71] G. Wentzel, Eine Verallgemeinerung der Quantenbedingungen für die Zwecke der Wellenmechanik, Z. Phys. 38(6), 518-529, (1926).

[72] H. A. Kramers, Wellenmechanik und halbzahlige Quantisierung, Z. Phys. 39(10), 828-840, (1926)

[73] L. Brillouin, La mécanique ondulatoire de Schrödinger; une méthode générale de resolution par approximations successives, Compt. Rend. Hebd. Seances Acad. Sci. 183(1), 24-26, (1926).

[74] L. D. Landau and E. M. Lifshitz, Statistical Physics, Part 1. vol. 5, Course of Theoretical Physics, (Butterworth-Heinemann, Oxford, 1980). ISBN 9780750633727.

[75] T. Asaka, M. Laine, and M. Shaposhnikov, On the hadronic contribution to sterile neutrino production, JHEP. 06, 053, (2006). 